AJSRP

ISSN: 2518- 5780
المجلة العربية للعلوم

ونشـر الأبحـاث

المجلد (7)، العدد (3): 30 سبتمبر 2021 م

ص: 75 - 93

\title{
Social adjustment and self- esteem among a sample of visitors and non- visitors to the psychological clinic after being exposed to psychological trauma (Sexual harassment as an example)
}

\author{
Zahraa Hussain Almosawi \\ Lebanon University || Lebanon
}

Ahmad Fadhel Alkout

Mohammad Abd Alali Akbar

Ministry of Education || Kuwait

\begin{abstract}
The study aims to investigate the differences between visitors and non- visitors to the psychological clinic after being exposed to psychological trauma (sexual harassment as an example) in Social adjustment and self- esteem, as well as to discover the relationship between study variables among a purposive sample consisted of 54 participants (17=visitors, 37=non- visitors), 31.4\% were males. To achieve the study aims two scales were used: Social Adjustment scale by "Sura, E. M." (1986), and Self- esteem scale by "Rosenberg, M." (1965). Results revealed that there were significant differences between visitors and non- visitors to psychological clinic after being traumatized in social adjustment and self- esteem. In addition, the results showed significant correlation between social adjustment and self- esteem among the total sample, visitors and non- visitors of the psychological clinic. The full text of the study includes details of the results and discussion, as well as some of recommendations made by the researchers.
\end{abstract}

Keywords: Social adjustment, self- esteem, psychological clinic, psychological trauma, sexual harassment.

$$
\begin{aligned}
& \text { التوافق الاجتماعي وتقدير الذات لدى المراجعين وغير المراجعين للعيادة النفسية } \\
& \text { بعد التعرض للصيدمة النفسية (التحرش الجنسي نموذجاً) } \\
& \text { زهراء حسين الموسوي } \\
& \text { |الجامعة اللبنانية || لبنان } \\
& \text { أحمد فاضل الكوت } \\
& \text { محمد عبد العلي أكبر } \\
& \text { وزارة التربية || الكويت }
\end{aligned}
$$

المستخلص: هدفت الدراسة الحالية لاستكشاف الفروق بين المراجعين وغير المراجعين للعيادة النفسية بعد التعرض للصددمة النفسية (التحرش الجنسي) في التوافق الاجتماعي وتقدير الذات، وكذلك للكشف عن العلاقة بين متغيريّ الدراسة، وذلك على عينة قصدية

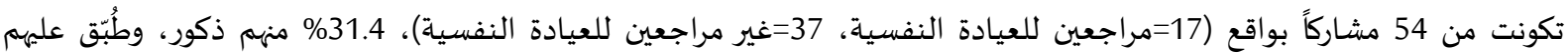
مقياسي التوافق الاجتماعي من إعداد (سرى، 1986)، وتقدير الذات (Rosenberg, M. 1965). وكشفت نتائج الدراسـة عن وجود فروقاً جوهرية بين المراجعين وغير المراجعين للعيادة النفسية بعد التعرض لصدمة التحرش الجنسي في كل من التوافق الاجتماعي وتقدير 
الذات وذلك لصالح المراجعين، وكذلك وجدت علاقة ارتباطية دالة بين متغيريّ الدراسة لدى العينة الكلية والمراجعين وغير المراجعين

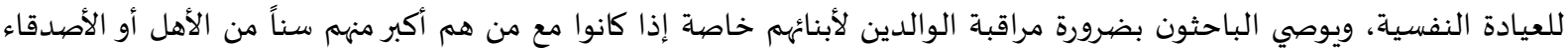

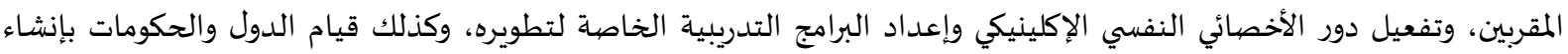

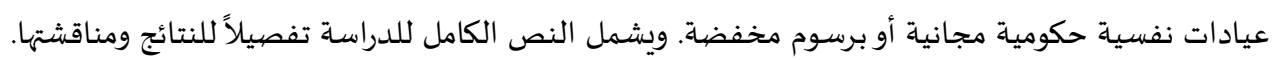
الكلمات المفتاحية: التوافق الاجتماعي، تقدير الذات، العيادة النفسية، الصدمة النفسية، التحرش الجنسي.

المقدمة.

يرتبط التعرض لأحداث الحياة المؤلمة بزيادة خطر الإصابة بالصدمات والاضطرابات النفسية كالتفكير في

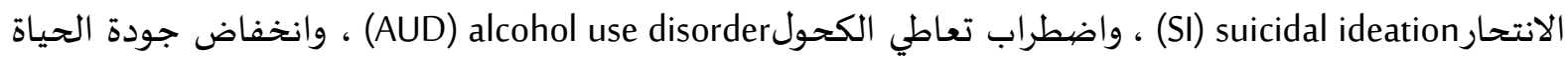

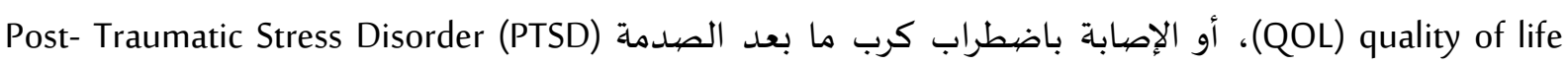
(Kachadourian et al. 2021)

ويذكر الدليل التشخيصي الاحصائي الخامس للاضطرابات النفسية أن التعرض للتحرش أو الإيذاء الجنسي .

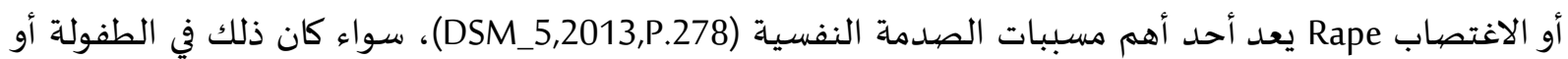
ما بعدها. وفي هذا الصدد حاول الكثير من علماء النفس والمعالجين والاختصاصييين النفسيين ابتكار طرق علاجية

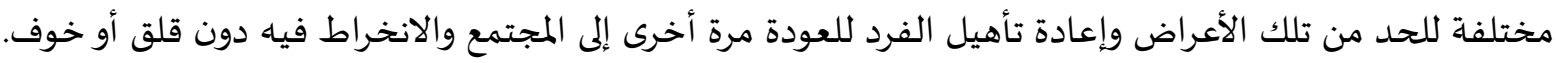

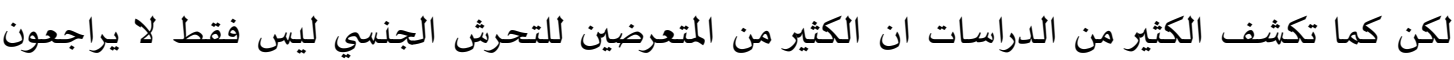

لطلب الاستشارة النفسية، بل انهم لا يتحدثون عن هذا الحدث مع أي شخص آخر (British Crime Survey,2002)

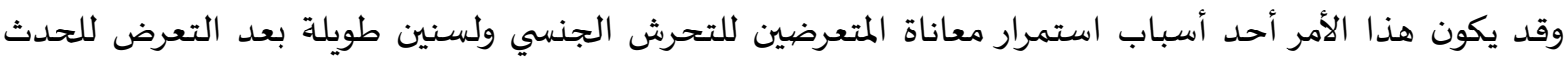
الصادم.

ويحاول الباحثون في هذه الدراسة التطرق إلى أثر مراجعة العيادات النفسية لتلقي العلاج النفسي بأشكاله

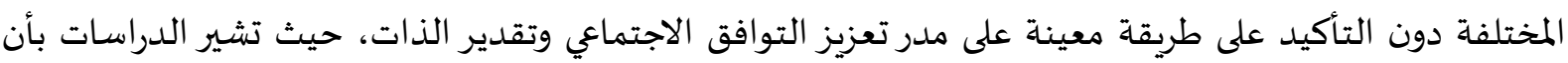

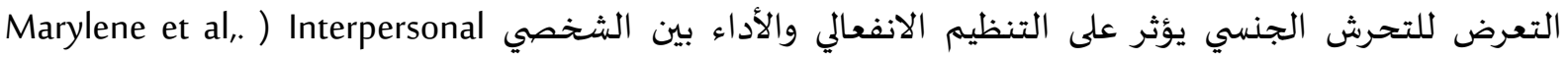
2005)، كما بينت بعض الدراسات أن التعرض للتحرش الجنسي قد يخلق مشكلة أخرى لدى الفرد وهي مشكلة

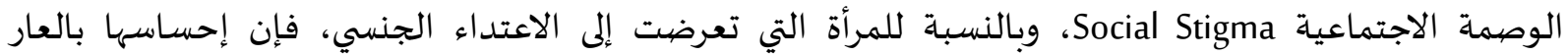

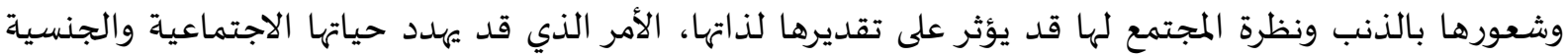

.(Finkelhor \& Browne's.1985)

مشكلة الدراسة:

تتحدد مشكلة هذه الدراسة في التحقق من أثر المراجعة للاستشارة النفسية بعد التعرض للصيدمة النفسية

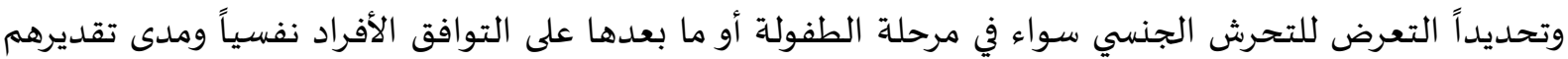
لذواتهم. فالتعرض للصدمة النفسية وخصوصاً صدمة التحرش الجنسي قد يترتب عليها تعديل وتغيير في السلوكيات والحالة النفسية والأفكار تجاه الذات والآخر والمستقبل والحياة الاجتماعية (2012, Monson et al).

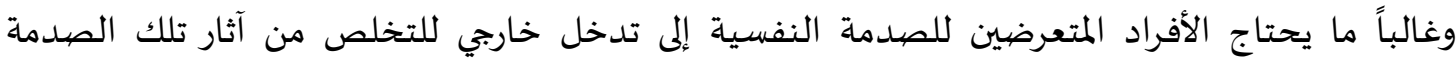
(Chivers- Wilson,2006). وهذا الأمر ليس بالجديد، فقد كان الناس قديماً يراجعون الحكيم أو الساحر أو العالِم

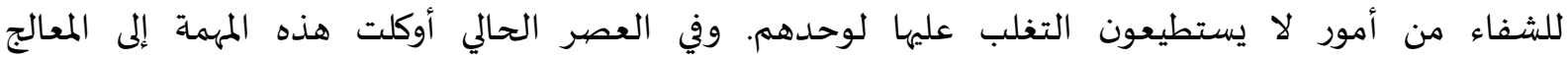
النفسي Psychologist أو الاستشاري النفسي Psychological consultant، وفي الحالات الحادة التي يصاب بها الفرد 
بالقلق أو الاكتئاب الشديد بسبب الصبدمة النفسية، قد يحتاج إلى التدخل الدوائي عن طريق المراجعة للطبيب

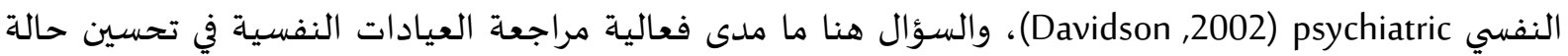
الفرد المزاجية وخفض أعراض الاضطرابات النفسية وتحقيق الأهداف المنشودة، هذا ما تسعى الدراسة الحالية للكشف عنه، حيث يمكن تلخيص مشكلة الدراسة في التساؤلات التالية:

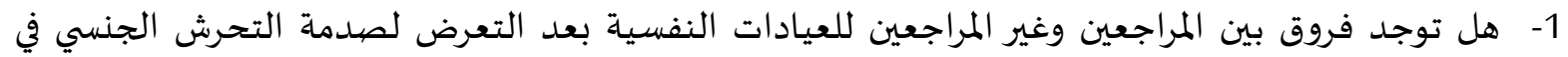

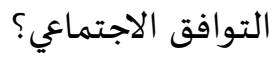
2- هل توجد فروق بين المراجعين وغير المراجعين للعيادات النفسية بعد التعرض لصدمة التحرش الجنسي في

$$
\text { تقدير الذات؟ }
$$

3- هل توجد علاقة ارتباطية بين التوافق الاجتماعي وتقدير الذات لدى المراجعين وغير المراجعين للعيادة النفسية بعد التعرض لصبدمة التحرش الجنسي والعينة الكلية؟

فرضيات الدراسة:

بعد مراجعة الدراسات السابقة والإطار النظري وبناءً على تصورات الباحثين، تمت صياغة الفرضيات

1- توجد فروق بين المراجعين وغير المراجعين للعيادات النفسية بعد التعرض لصيدمة التحرش الجنسي في

$$
\text { التوافق الاجتماعي. }
$$

2- توجد فروق بين المراجعين وغير المراجعين للعيادات النفسية بعد التعرض لصدمة التحرش الجنسي في تقدير

3- توجد علاقة ارتباطية بين التوافق الاجتماعي وتقدير الذات لدى العينة الكلية والمراجعين وغير المراجعين

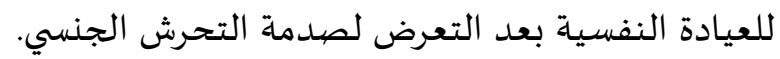

أهداف الدراسـة

إن اتخاذ القرار لمراجعة المعالج أو الطبيب النفسي يعتبر من القرارات الصعبة للأفراد في ظل وجود وصمة

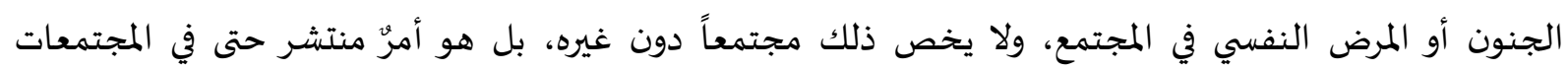
الصناعية والمتطورة (Johnson- Kwochka,2021).

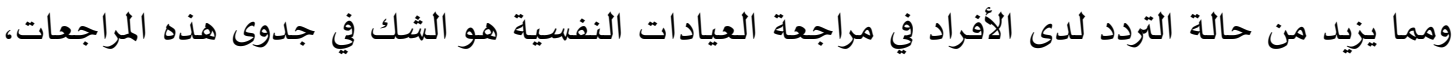

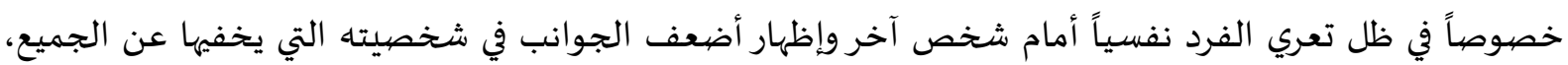

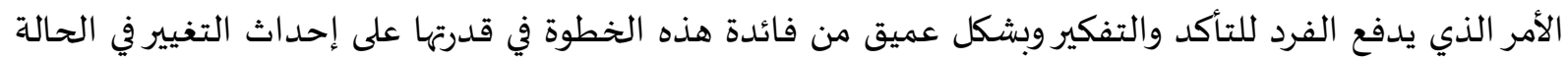

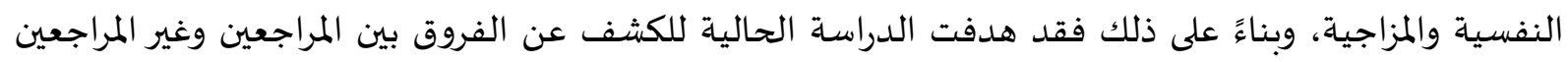

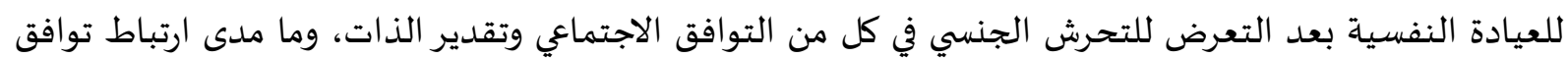

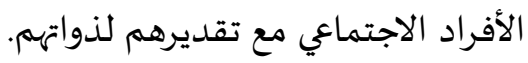

أهمية الدراسة:

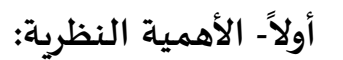
1- تعد نتائج هذه الدراسة إثراءً علمياً حول الاثار النفسية للتعرض للتحرش الجنسي وأثر معالجتها أو ترك الصيدمة كما هي دون علاج. 
2- توفر هذه الدراسة بعض المعلومات التي قد يستفيد منها المعالجين والاخصائيين النفسيين في علاج مراجعيهم الذين تعرضوا لصهدمة التحرش الجنسي.

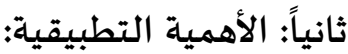

1- تكمن أهمية هذه الدراسة في ندرة الدراسات التي تتطرق لجدوى المراجعة لمراكز الاستشارة للتخلص من اثار الصدمة وتحديداً صدمة التحرش الجنسي. حيث اقتصرت الدراسات السابقة غالباً على درجة تأثير برامج علاجية معينة يتم تصميمها وتنفيذها على مجموعة صغيرة من المشاركين وهذه الدراسات غالباً ما تبين الأثر الإيجابي وذلك لأسباب مختلفة. لكن تخصيص دراسة ترصد هذا الأثر بشكل عام وبغض النظر عند عن وهن نوه

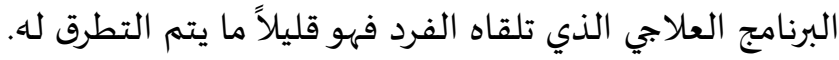
2- تساعد هذه الدراسة المتعرضين للتحرش الجنسي في اتخاذ القرار لمراجعة المختص للتخلص من آثار الصدمةهة، حيث قد تطول عملية اتخاذ القرارعدة سنوات بسبب نقص المعرفة وعدم التأكد من جدوى المراجعة.

حدود الدراسة:

شملت الدراسة مجموعة من المتعرضين للتحرش الجنسي الذين راجعوا العيادات النفسية للتخلص من آثار الصيدمة من العينة القصدية المتاحة لدى الباحث، وذلك لصعوبة الوصول لمثل هذه العينة في المراكز أو المؤسسات

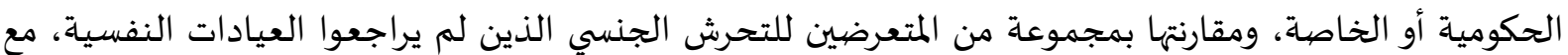
مراعاة ضبط المتغيرات التي من شأهها أن تؤثر في نتيجة الدراسة كالجنس والعمر والحالة الاجتماعية والبلد، وتم

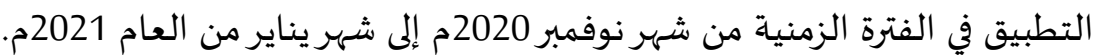

2- الإطار النظري والدراسـات السـابقة. الصدمة النفسية هي حدث أو تجربة يمر بها الفرد وتؤدي خلال فترة وجيزة لزيادة كبيرة من الإثارات

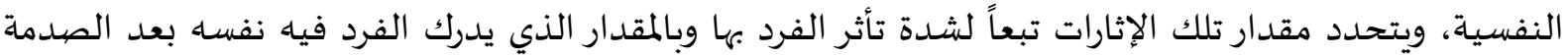

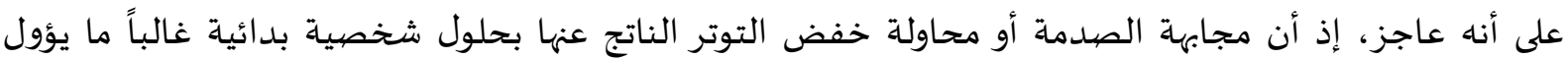

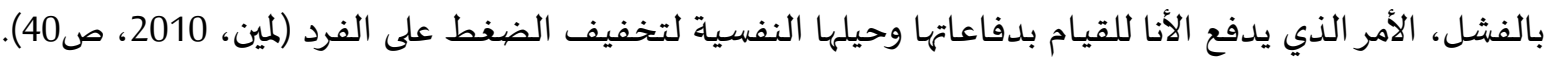

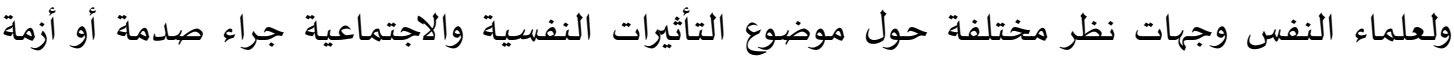

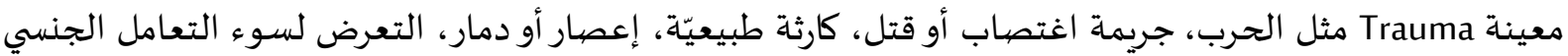
أو العاطفي في الطفولة أو فقدان شخص عزيز بطريقة كارثية، ويختلف تفسير العلماء لأسباب السلوكيات

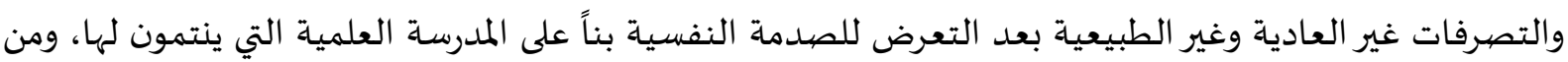
أهم تلك التوجهات:

مدرسة التحليل النفسي: فبحسب اوتو رانك (1924) فان الصلدمة الأولى التي يتعرض لها الشخص هي صدمة الولادة (Pizarro,2012)، ويفسر Freud الصدمة بأهها تجربة يعيشها الفرد وتسبب له خله خلال وقتاً قصيراً نسبيا

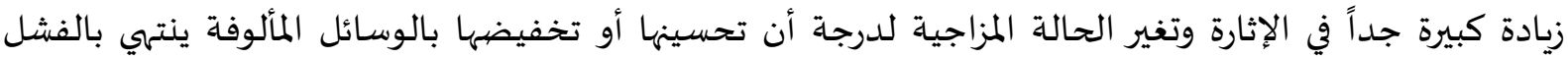

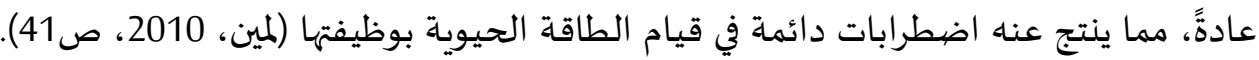

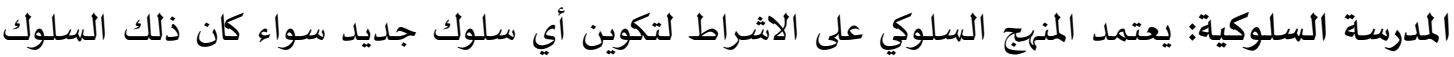

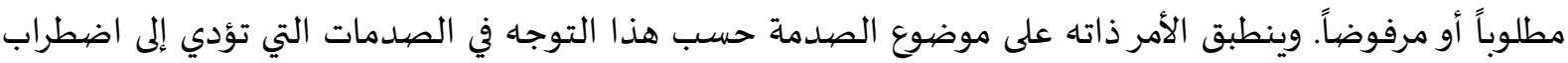
كرب ما بعد الصيدمة PTSD فإن الاشراط الكلاسيكي، خاصةً في ظرف وجود حادث صادم، قد يتسبب في اكساب 
الفرد استجابة خوف شرطية لتنبيه طبيعي (مثير غير شرطيّ)، فالمرأة- على سبيل المثال- التي كانت قد تعرضت إلى

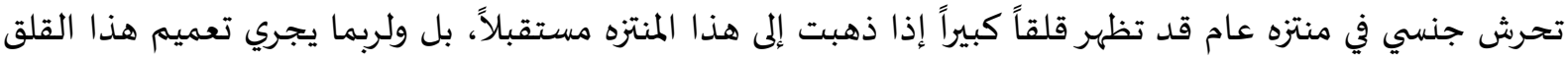

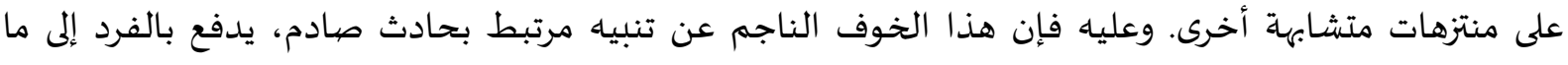

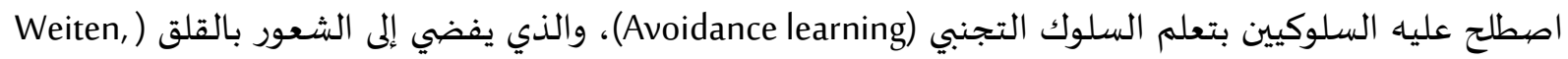

المدرسة المعرفية: وترى هذه المدرسة أن الاحداث الصادمة تهدد افتراضاتنا العادية أو السوية بخصوص

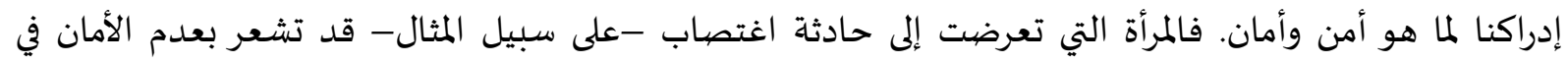

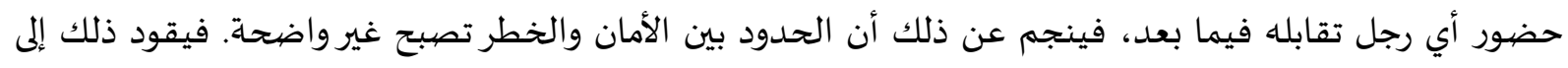

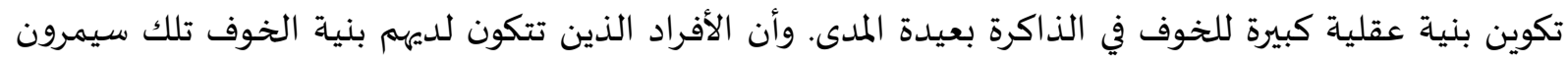

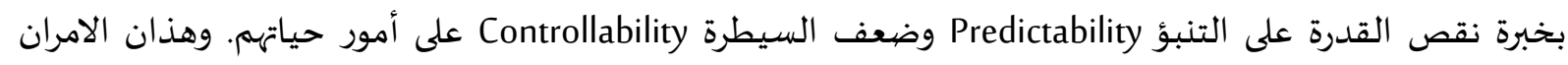

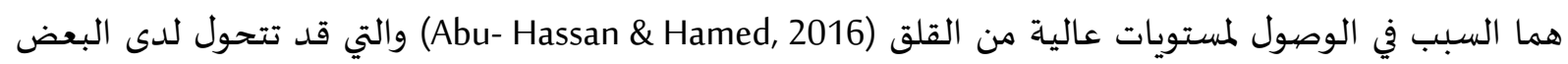
إلى اضطراب كرب ما بعد الصيدمة. ويعرف (Haviland et al 2016) اضطراب ما بعد الصدمة بأنه حالة نفسية خطيرة قد تتبع حدثًا مؤلمًا يتميز

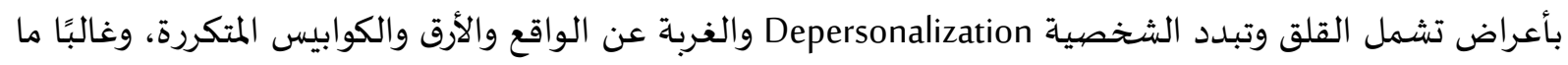
تكون هذه الأمور منهكة لدرجة أن العمل العادي والأنشطة الاجتماعية تكون صعبة أو أو مستحيلة.

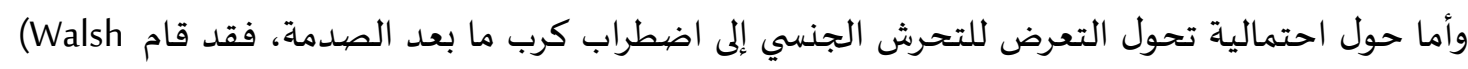

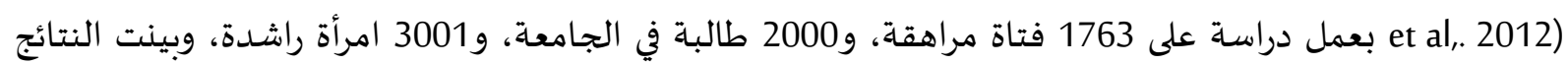
بأن أكثر من 50\% من الاناث في كل مجموعة من هذه المجموعات الثلاث تعرضن للإيذاء الجنسي بأشكاله المختلفة.

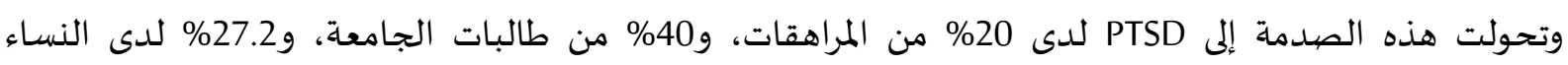

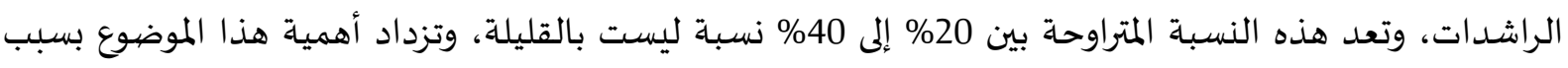

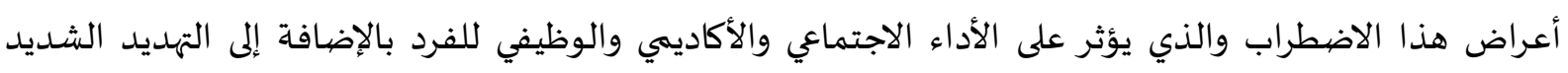
لصحتا النفسية.

أما (خفاجي، 2008) فيرى أن التحرش الجنسي سلوك غير مرغوب فياء يتم بدون موافقة الضحية ويشمل

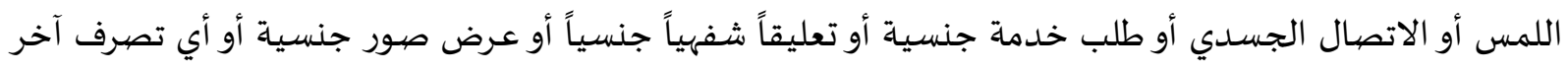

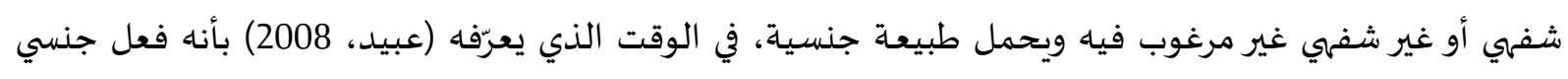

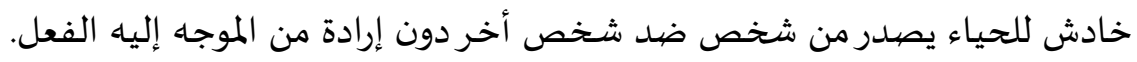

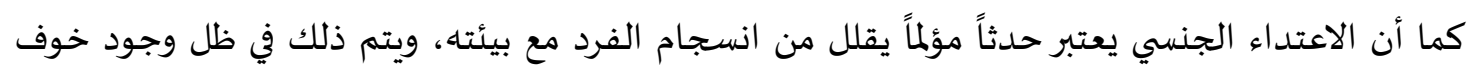

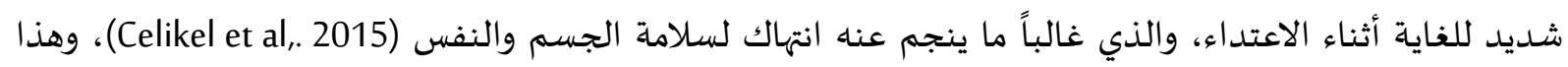

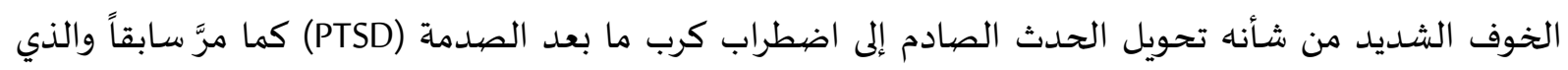

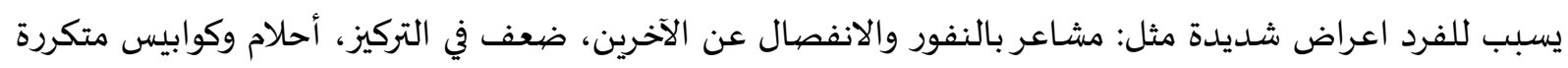

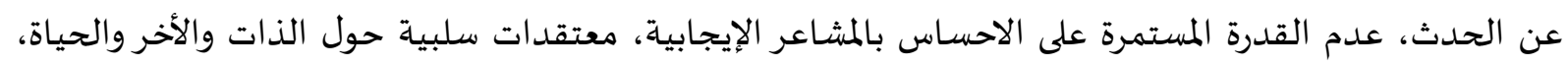
التهور أو السلوك التدميري للذات وأعراض أخرى مؤلمة (DSM 5,2013,P 271). ولعلاج هؤلاء المصابين بالصدمة النفسية، استخدم المعالجين النفسيين والاستشاريين طرق علاجية مختلفة منها الأساليب العامة التي تستخدم في علاج كثير من الاضطرابات النفسية مثل العلاج المعرفي السلوكي والعلاج 
الجشتالطتي وعلاج المخططات المعرفية غير التكيفية الأولية، وتم كذلك استخدام تقنيات خاصية باضطراب كرب ما بعد الصدماة مثل البرامج التالية:

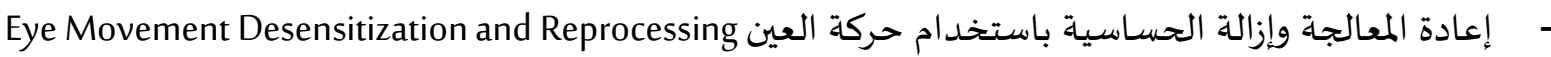
(EMDR)

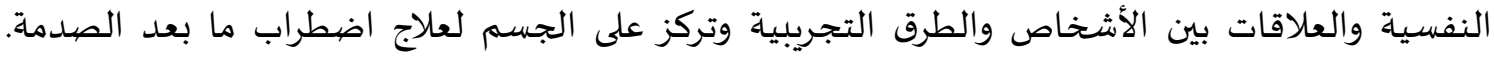

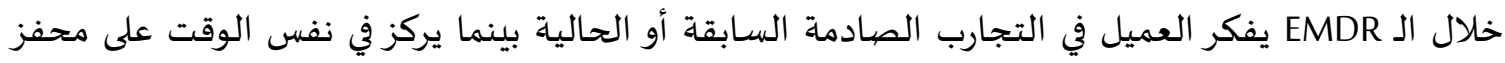
مثل النغمات السمعية أو التحفيز اللمسي أو الإشارات البصرية (Shapiro,2002).

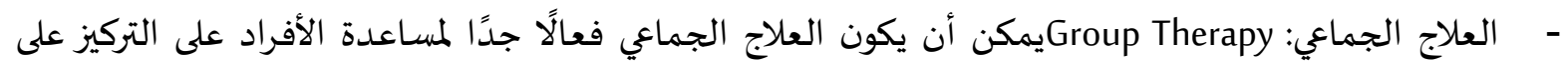

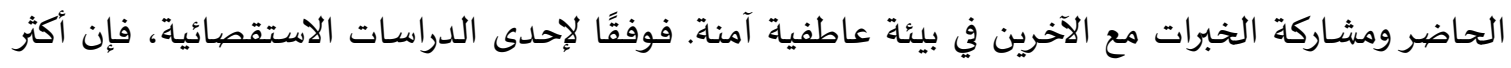

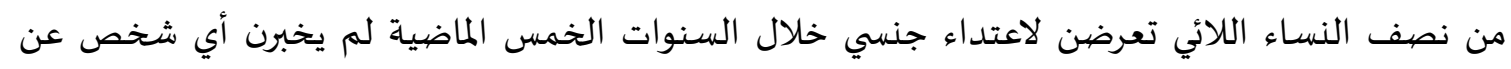

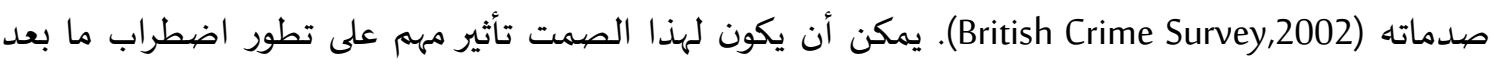
الصدمة، حيث إن الدعم والمساندة الاجتماعية قد تخفف من شدة الأعراض (Dunmore, Ehlers,1999)

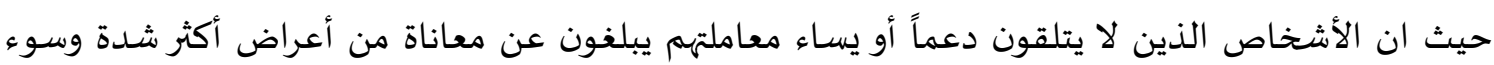

(Koss \& Figuerdo, 2004)

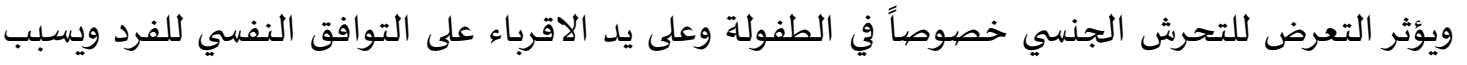

له الكثير من المشاكل النفسية والاجتماعية (1999, Roche et al).

ويشمل التوافق النفسي أربعة ابعاد أساسية هي عبارة عن: التوافق الاجتماعي، التوافق الشخصيه الشئ، التوافق

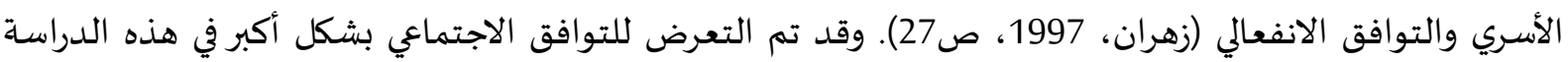

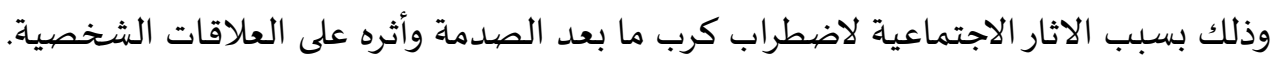

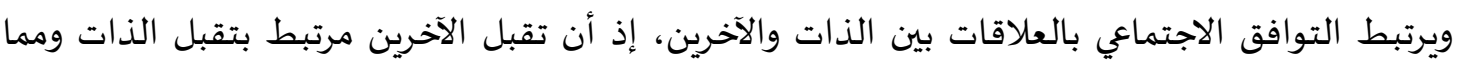

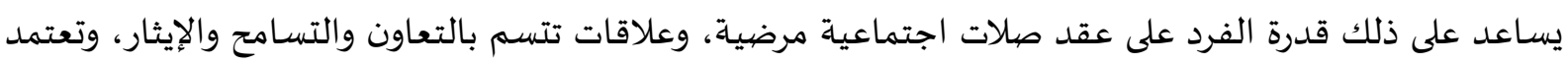

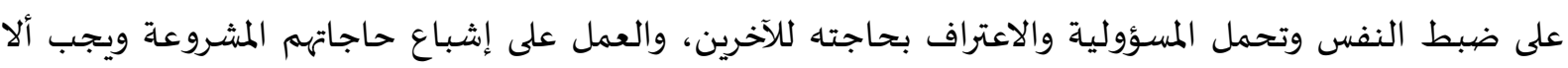

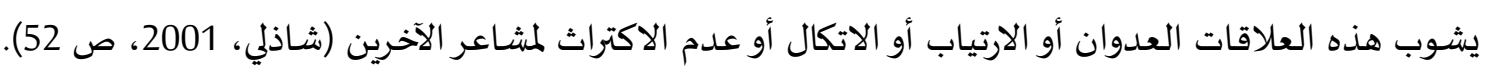

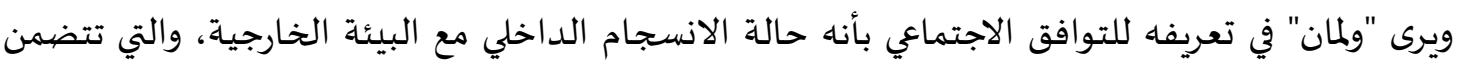

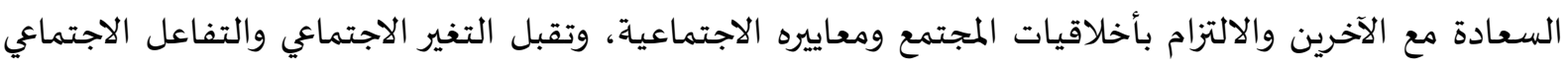

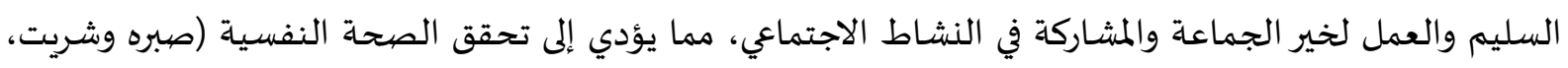
2004، ص 125).

وبينت بعض الدراسات أن التعرض لصددمة التحرش الجنسي تثير بعض المشـاعر والأفكار والسلوكيات التي

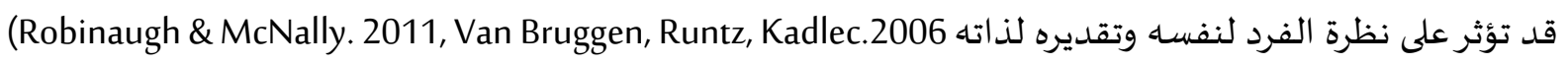
)، وينقسم تقدير الذات Self- esteem إلى نوعين أساسيين وهما:

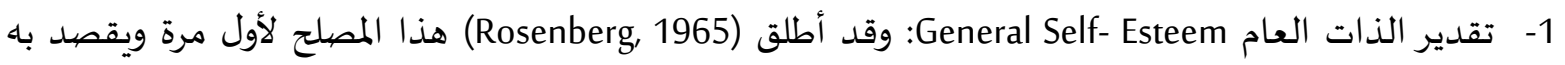

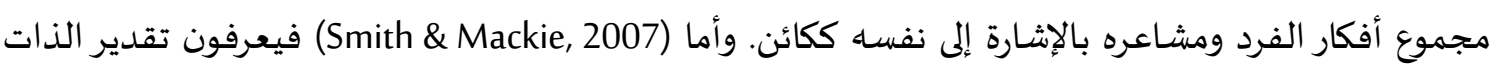

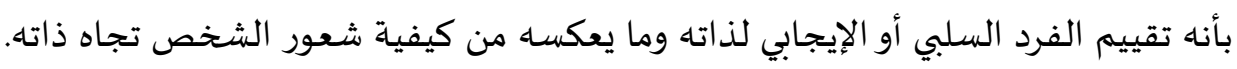

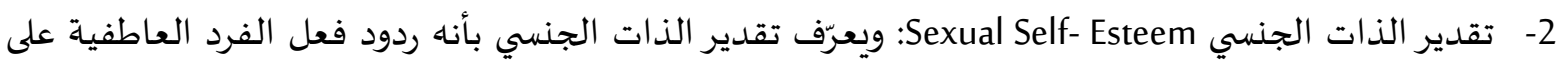

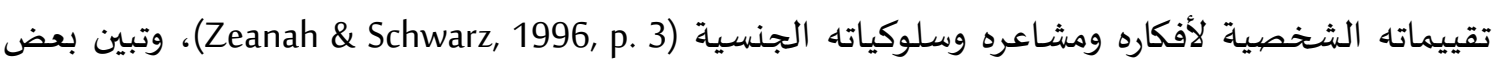


(Oattes \& Offman, الدراسات بأن هذان النوعان مرتبطان ببعض ويؤثر أحدهما على الآخر كما ظهر في دراسة

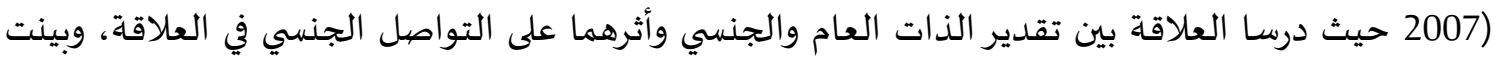

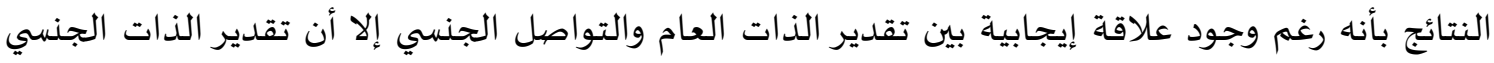

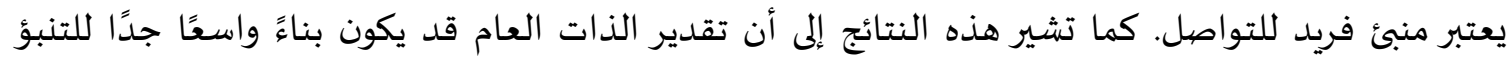
بقدرة الفرد على مناقشّة الاحتياجات الجنسية مع الشريك. ويعرف معدوا هذه الدراسة التوافق الاجتماعي وتقدير الذات اجرائياً: بأنها الدرجة التي يحصل التهل عليها المفحوصين في مقياسي التوافق الاجتماعي وتقدير الذات.

ثانياً- الدراسات السابقة: لاقت الصدمة النفسية الناتجة عن التحرش الجنسي اهتماماً من المختصيين النفسيين والاجتماعيين وذلك الماتك

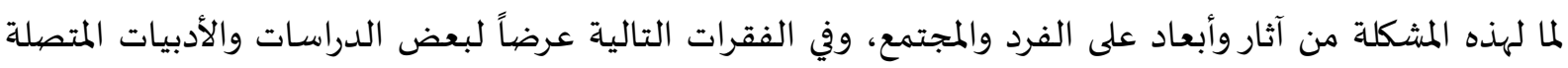

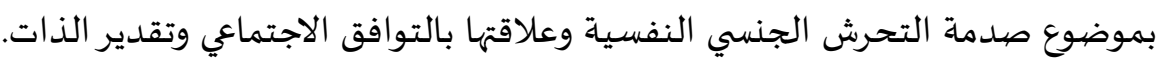

أ- الدراسات التي ربطت بين صدمة التحرش الجنسي والتوافق الاجتماعي.

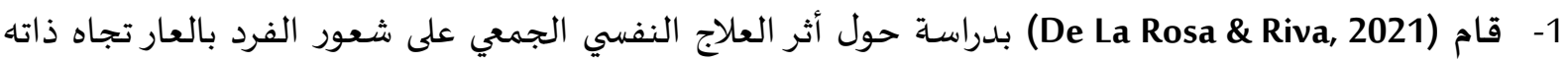
واضطراب كرب ما بعد الصدمة وذلك على عينة تكونت من 24 فتاة بالغة و9 فتيات من الأطفال المتعرضين آتراني

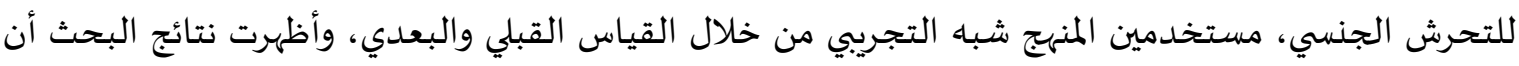

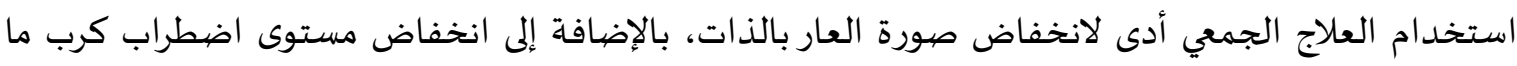

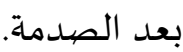
2- كذلك قام (Wright \& Warner. 2020) بدراسة أثر العلاج النفسي على مجموعة من الأطفال الذين تعرضوا

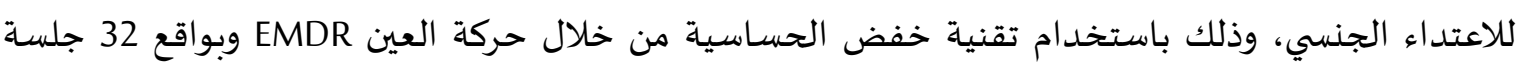

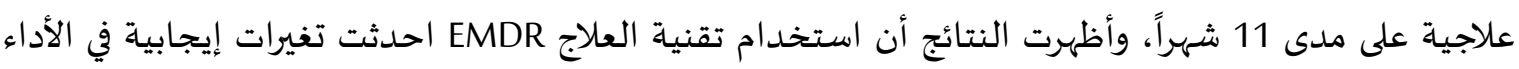
العاطفي والمعرفي والفسيولوجي للمتعرضين للتحرش الجنسي. 3- أجرى (Kucharska, 2017) دراسة تهدف للكشف عن الآثار النفسية للمتعرضين للصيدمة الجنسية مقارنةً بغير

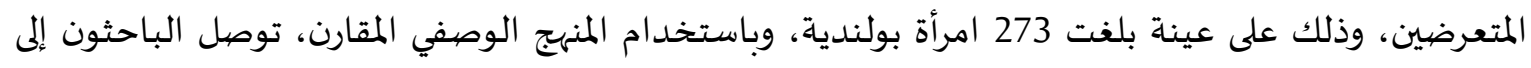

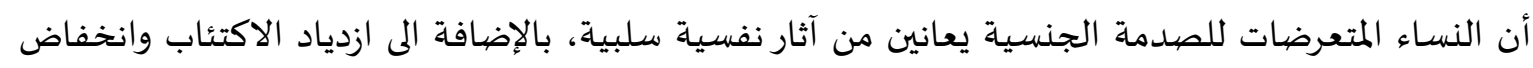
تقدير الذات مما يتسبب بمشاكل عدم التوافق والانسجام. 4- أما (Jaberghaderi et al,. 2004) فقد قاموا بدراسة لمعرفة أثر فاعلية العلاج السلوكي المعرفي CBT مقارنةً بعلاج خفض الحساسية بحركة العينEMDR، حيث استخدموا المنهج شبه التجريبي على عينة عددها 14 فتاة إيرانية

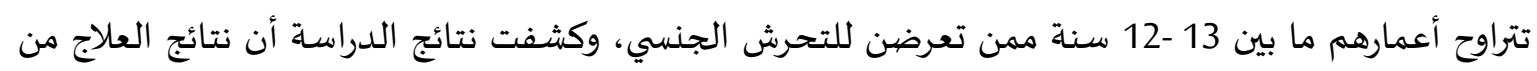

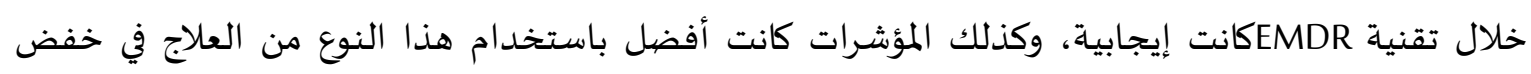
مستوى الصدمة للمتعرضين للتحرش الجنسي. ب- الدراسات التي ربطت بين صدمة التحرش الجنسي وتقدير الذات.

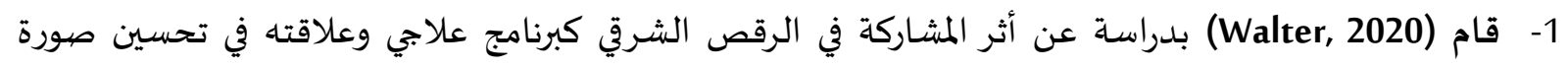

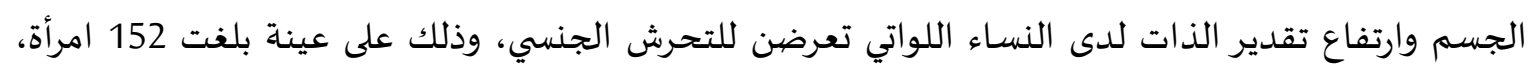


وكشفت نتائج الدراسـة عن وجود ارتباط إيجابي وجوهري بين الرقص الشرقي وتقدير الذات وصورة الجسم لدى النساء المتعرضات للتحرش الجنسي. 2- أما (Barnum \& Perrone- McGovern, 2017) فقد قاموا بدراسة تهدف الى الكشف عن العلاقة بين الصدمات الجنسية وتقدير الذات والهناء الشخصي Well- being وذلك على عينة تكونت من 213 طالبا جامعيا

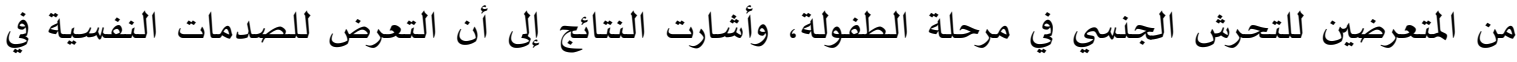

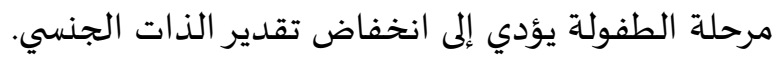
3- وكذلك قام (Pretorius \& Pfeifer, 2010) بدراسة على عينة تكونت 25 فتاة تتراوح أعمارهن بين (8- 11) سنة، وذلك لبحث أثر العلاج الجمعي على الفتيات اللاتي تعرضن للتحرش الجنسي، ومدى فاعلية هذا النوع من العلاج في خفض أعراض الاكتئاب والقلق والصدمات الجنسية وانخفاض تقدير الذات، وذلك باستخدام المنهج

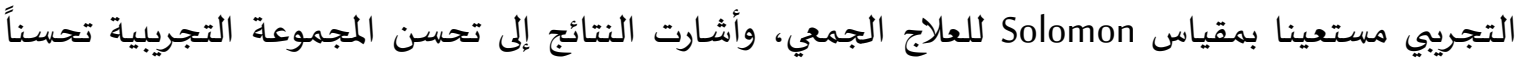

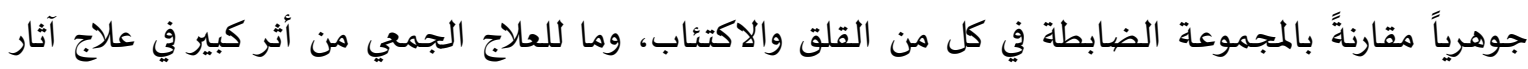
التحرش والايذاء الجنسي على الأطفال.

4- أما (Westbury \& Tutty, 1999) فقد قاموا بدراسة أثر العلاج الجمعي للمتعرضين للاعتداء الجنسي وعلاقته في رفع مستوى تقدير الذات وخفض مستوى الاكتئاب والقلق، وذلك على عينة من 32 امرأة، باستخدام المنهنج شبه التجريبي لقياس الفروق بين المجموعتين، وكشفت النتائج عن أن العلاج الجمعي أكثر فعالية من العلاج الفردي في رفع تقدير الذات وخفض مستوى القلق والاكتئاب.

$$
\text { التعقيب على الدراسات السابقة: }
$$

1- تؤثر مراجعة العيادة النفسية في تحسن الصحة النفسية بعد التعرض لصيدمة التحرش الجنسي.

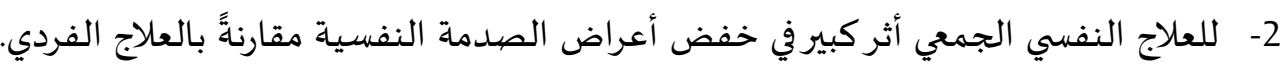

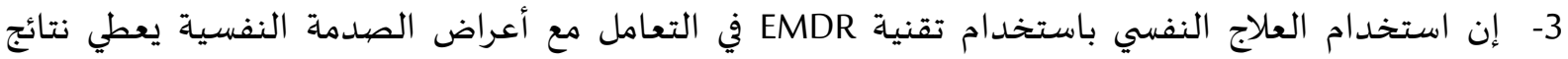
أفضل مقارنةً ببقية العلاجات. 4- - قد يكون لبعض أنواع العلاج النفسي القائم على النشاط البدني من خلال ممارسة بعض التمات التمارين والحركات

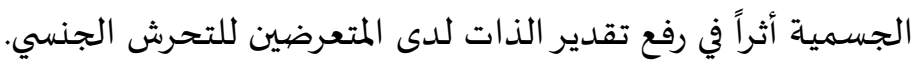

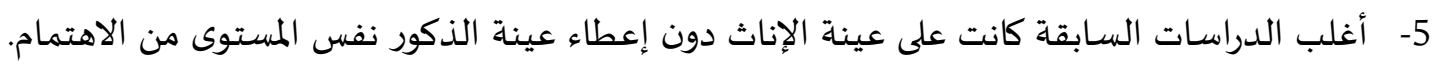

3- منهجية الدراسـة وإجراءاتها.

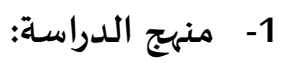

استخدم الباحثون المنهج الوصفي (التصيميم الارتباطي المقارن) للمقارنة بين المراجعين وغير المراجعين

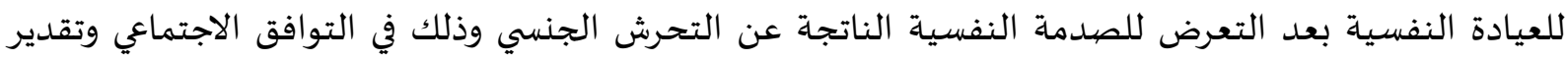
الذات، وكذلك للكشف عن العلاقة الارتباطية بين متغيريّ الدراسـة.

2- 2 - خصيائص عينة الدراسة: أ- العينة الاستطلاعية: قام الباحثون بالتأكد من الخصائص السيكومترية للمقاييس المستخدمة، والتحقق من صلاحية تلك الأدوات لأهداف الدراسة، وذلك على عينة استطلاعية تكونت من (20) مشاركاً من الجنسين، 
راجع نصفهم العيادة النفسية، وتراوحت أعمارهم بين (18- 34) عاماً، بمتوسط عمري بلغ 25.2 عاماً،

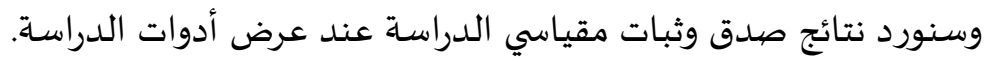

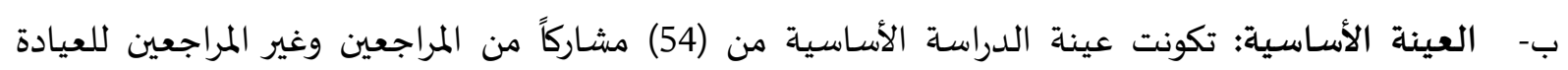
النفسية بعد التعرض لصيدمة التحرش الجنسي ومن الجنسين وذلك باستخدام أسلوب العينة القصيدية

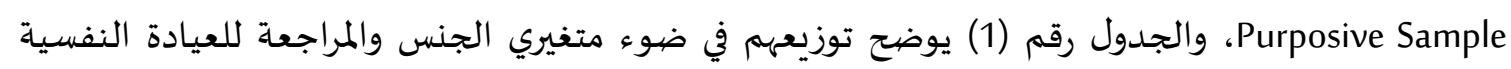
على النحو التالي:

جدول رقم (1) لتوزيع أفراد عينة الدراسة حسب الجنس والمراجعة للعيادة النفسية

\begin{tabular}{|c|c|c|c|}
\hline 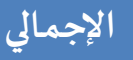 & إناث اث & 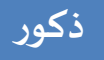 & وجه التوزيع \\
\hline 17 & 15 & 2 & المراجعين للعيادة النفسية \\
\hline 37 & 22 & 15 & غير المراجعين للعيادة النفسية \\
\hline 54 & 37 & 17 & الإجمالي \\
\hline
\end{tabular}

$$
\text { 3- أد مقياس التوافق الدراسة: }
$$

يتكون مقياس التوافق النفسي في صورته الأساسية من 40 فقرة تقيس أربعة أبعاعي، أبعاد من التوافق وهي:

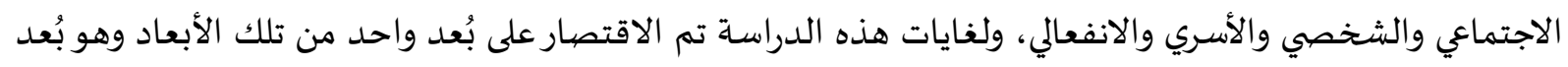

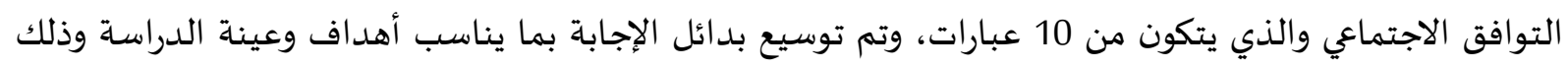

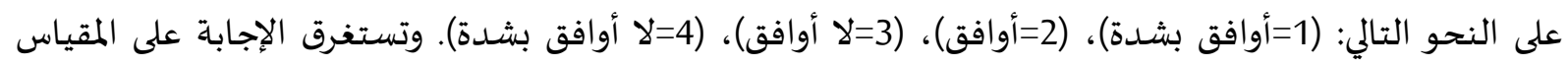

أقل من خمسة دقائق.

وقام الباحثون بحساب ثبات الاختبار بطريقة ألفا- كرونباخ وكذلك طريقة التجزئة النصفية باستخدام

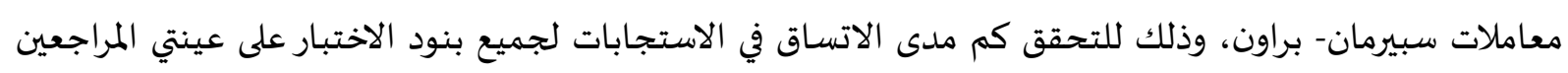

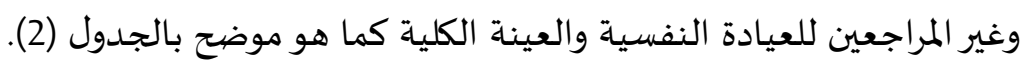

جدول (2) لمعاملات ثبات مقياس التوافق الاجتماعي على عينتي المراجعين وغير المراجعين للعيادة النفسية

\begin{tabular}{|c|c|c|c|c|}
\hline العينة الكلية & غير مراجعين للعيادة & النفسية (ن=عين للعيادة & 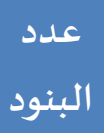 & طريقة الثبات \\
\hline 0.81 & 0.79 & 0.78 & \multirow{2}{*}{10} & معاملات ألفا \\
\hline 0.81 & 0.79 & 0.79 & & التجزئة النصفية \\
\hline
\end{tabular}

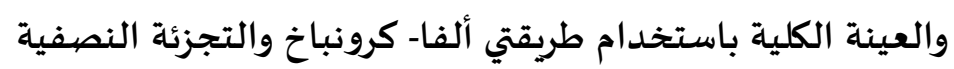

يتضح من الجدول (2) ارتفاع ثبات معامل ألفا- كرونباخ وكذلك التجزئة النصفية، وبالتالي تعتبر مقبولة في

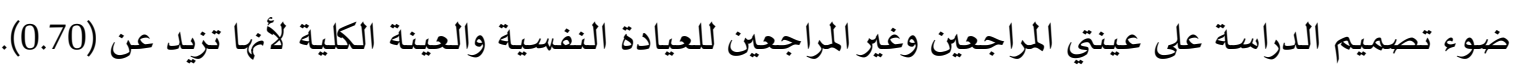

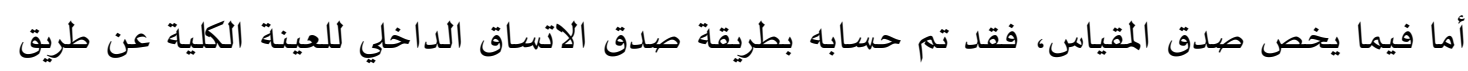

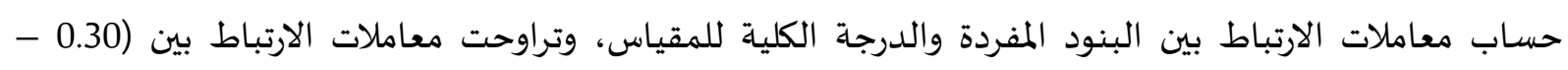

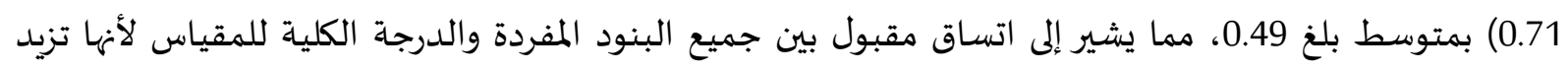
عن (0.30)، مما يدل على مناسبة هذا الاختبار لغايات هذه الدراسة. 
ب- مقياس تقدير الذات (Rosenberg, M. 1965):

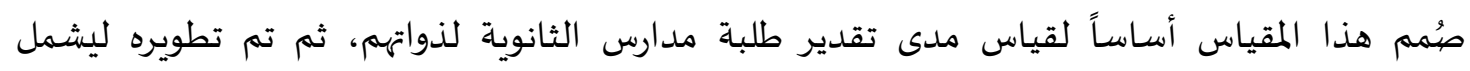

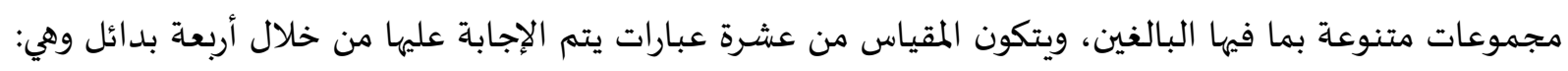

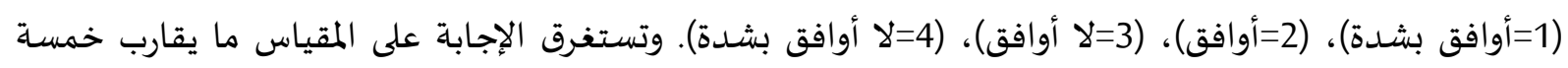

دقائق.

وقام معدوا هذه الدراسة بالتأكد من خصيائصها السيكومترية على عينة الدراسة، والتي كشفت عن حصول

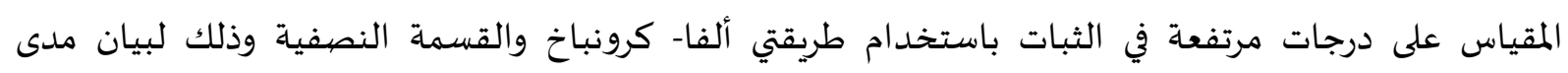

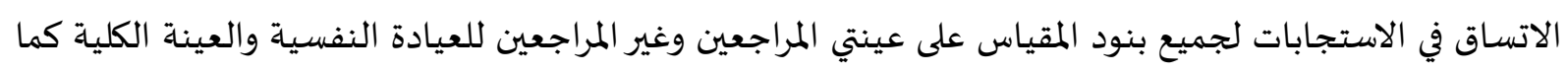

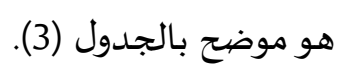

جدول (3) لمعاملات ثبات مقياس تقدير الذات على عينتي المراجعين وغير المراجعين للعيادة النفسية والعينة

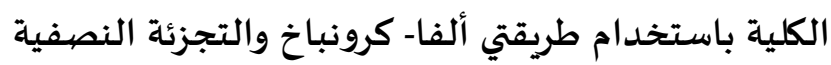

\begin{tabular}{|c|c|c|c|c|}
\hline العينة الكلية & غير مراجعين للعيادة & النفسية (ن=عين للعيادة & البنود عدد & طريقة الثبات العينة \\
\hline 0.86 & 0.85 & 0.81 & \multirow{2}{*}{10} & معاملات ألفا \\
\hline 0.88 & 0.84 & 0.90 & & التحزئة النصفية \\
\hline
\end{tabular}

يتضح من الجدول (3) ارتفاع ثبات معامل ألفا- كرونباخ وكذلك التجزئة النصفية، وبالتالي تعتبر مقبولة في

ضوء تصميم الدراسة على عينتي المراجعين وغير المراجعين للعيادة النفسية والعينة الكلية لأنها تزيد عن (3) (0.70).

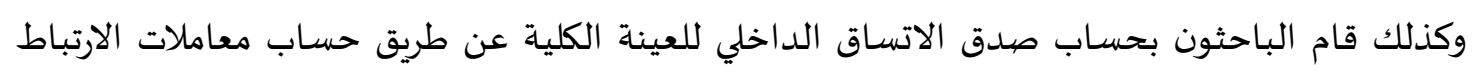

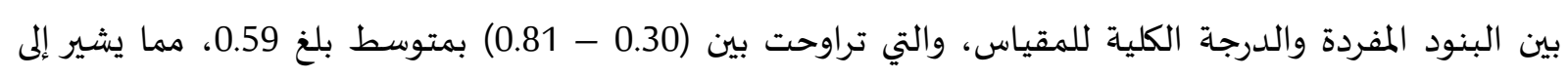

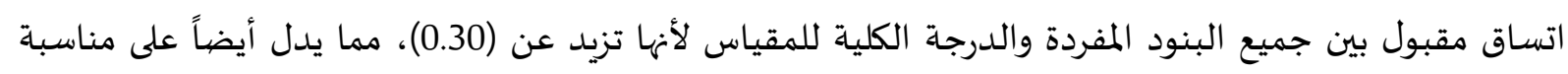
هذا الاختبار لغايات هذه الدراسة.

$$
\text { 4- الأساليب الإحصيائية: }
$$

استخدم الباحثون اختبار(ت) T- Test للتعرف على مدى الفروق ودلالتها بين المراجعين وغير المراجعين

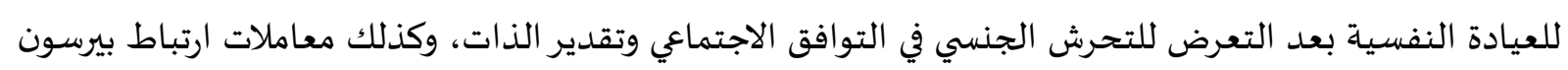
كearson Correlation

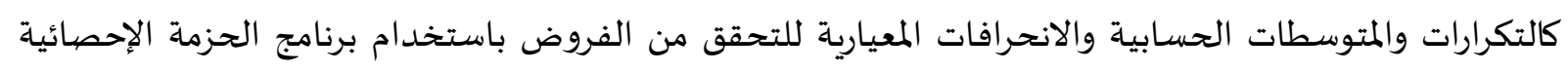
للعلوم الاجتماعية (SPSS). 
4- عرض نتائج الدراسـة ومناقشتها.

نتيجة الفرض الأول: "توجد فروق بين المراجعين وغير المراجعين للعيادة النفسية بعد التعرض لصدمة التحرش

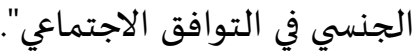

جدول (4) نتائج اختبار "ت" لدلالة الفروق بين متوسطات درجات المفحوصين المراجعين وغير المراجعين للعيادة النفسية بعد التعرض للتحرش الجنسي في مقياس التوافق الاجتماعي

\begin{tabular}{|c|c|c|c|c|c|c|c|c|}
\hline \multirow{2}{*}{ قريمة } & \multirow{2}{*}{ الدلالة } & \multirow{2}{*}{ "تيمة } & \multirow[b]{2}{*}{ ב. } & \multicolumn{2}{|c|}{ غير مراجعين للعيادة } & \multicolumn{2}{|c|}{ 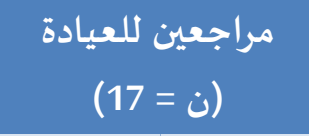 } & \multirow[t]{2}{*}{ المتغير } \\
\hline & & & & الانحراف & المتوسط & الانحراف & المتوسط & \\
\hline 0.81 & 0.014 & 2.54 & 52 & 5.1 & 19.8 & 5.1 & 23.6 & التوافق الاجتماعي \\
\hline
\end{tabular}

يتضح من الجدول (4) وجود فروقاً جوهرية بين المراجعين وغير المراجعين للعيادة النفسية بعد التعرض

للتحرش الجنسي في متغير التوافق الاجتماعي، حيث كانت متوسطات المراجعين أعلى من غير المراجعين للعيادة

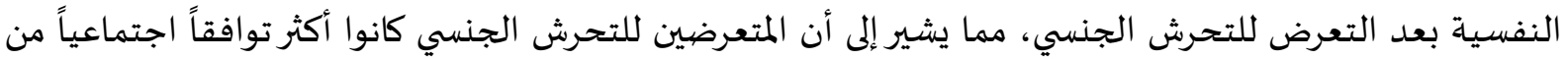

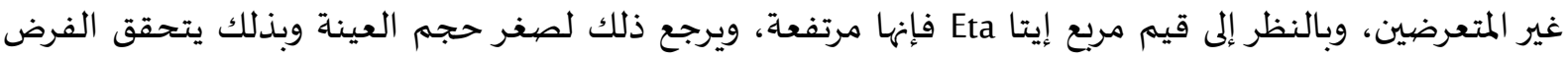

نتيجة الفرض الثاني: توجد فروق بين المراجعين وغير المراجعين للعيادة النفسية بعد التعرض لصدمة التحرش الجنسي في تقدير الذات.

جدول (5) نتائج اختبار "ت" لدلالة الفروق بين متوسطات درجات المفحوصين المراجعين وغير المراجعين للعيادة النفسية بعد التعرض للتحرش الجنسي في مقياس تقدير الذات

\begin{tabular}{|c|c|c|c|c|c|c|c|c|}
\hline \multirow{2}{*}{ قريمة } & \multirow{2}{*}{ مستوى الدلالة } & \multirow{2}{*}{ "تيمة } & \multirow[b]{2}{*}{ ני } & \multicolumn{2}{|c|}{ غير مراجعين للعيادة } & \multicolumn{2}{|c|}{$\begin{array}{c}\text { مراجعين للعيادة } \\
\text { (ن = 17 }\end{array}$} & \multirow{2}{*}{ المقياس / لمتغير } \\
\hline & & & & الانحراف & المتوسط & الانحراف & المتوسط & \\
\hline 0.89 & 0.001 & 3.56 & 52 & 5.1 & 19.0 & 5.2 & 24.4 & تقدير الذات \\
\hline
\end{tabular}

يتضح من الجدول (5) وجود فروقاً جوهرية بين المراجعين وغير المراجعين للعيادة النفسية بعد التعرض المرات

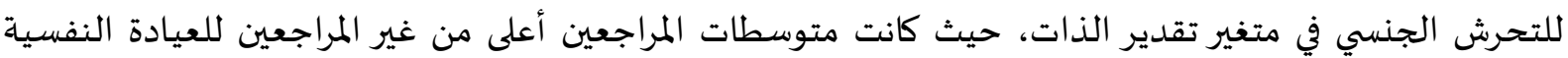

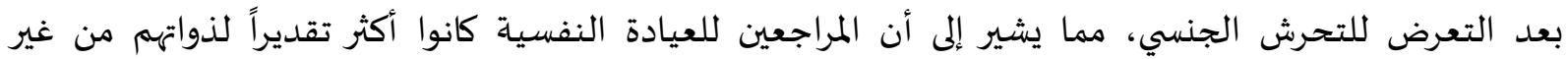

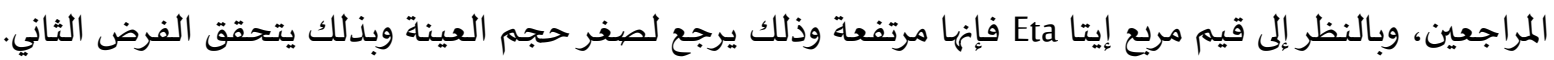

نتيجة الفرض الثالث: توجد علاقة ارتباطية بين التوافق الاجتماعي وتقدير الذات لدى العينة الكلية والمراجعين وغير المراجعين للعيادة النفسية بعد التعرض لصدمة التحرش الجنسي. 
جدول (6) للعلاقة الارتباطية بين التوافق الاجتماعي وتقدير الذات لدى العينة الكلية وعينتي المراجعين وغير

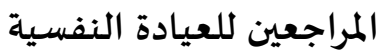

\begin{tabular}{|c|c|c|c|c|}
\hline \multirow[b]{2}{*}{ العينة الكلية (ن=54) } & \multicolumn{2}{|c|}{ تقدير الذات } & & \multirow[b]{2}{*}{ المتغيرات } \\
\hline & غير مراجعين للعيادة & النفسية (ن=-17) & & \\
\hline$* * 0.71$ & $* * 0.60$ & $* * 0.82$ & الارتباط & \multirow{2}{*}{ التوافق الاجتماعي } \\
\hline 0.001 & 0.001 & 0.001 & الدلالة & \\
\hline
\end{tabular}

0.01 معاملات ارتباط دالة عند مستوى

يتضح من الجدول (6) وجود علاقة ارتباطية إيجابية بين التوافق الاجتماعي وتقدير الذات وذلك على عينتي

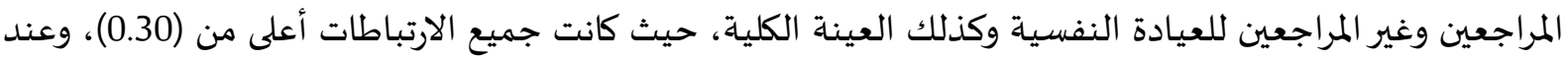
مستوى دلالة 0.01، وبالتالي تحقق الفرض الثالث.

مناقشـة نتائج الدراسـة.

مناقشَة الفرض الأول: توجد فروق بين المراجعين وغير المراجعين للعيادة النفسية بعد التعرض للتحرش الجنسي

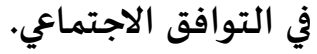

كشفت نتائج التحليل الاحصائي عن تحقق الفرض الأول، حيث تبين أن المراجعين للعيادة النفسية بعد

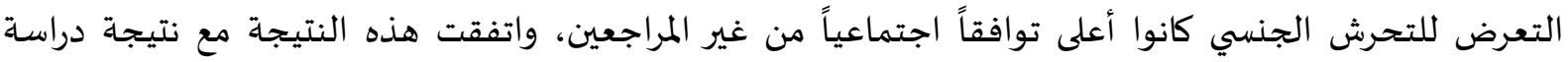
(Santarnecchi et al., 2019)

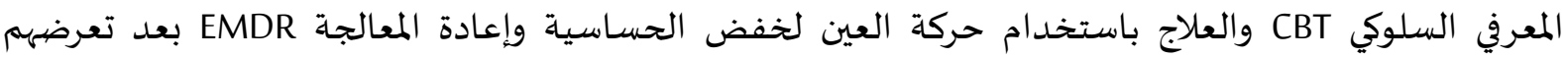

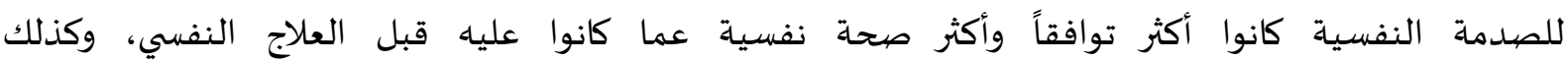
دراسة(Jaberghaderi et al., 2004) والتي بينت أن كلا الأسلوبين العلاجيين CBT \& EMDR يساهم في مساعدة النساء

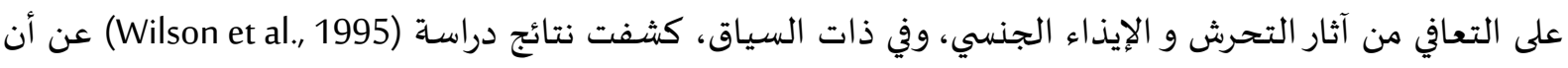

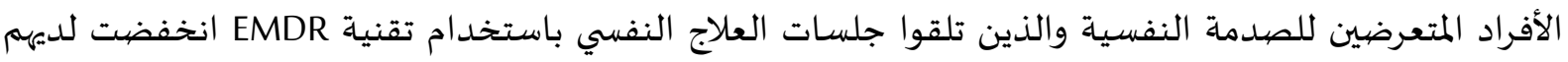

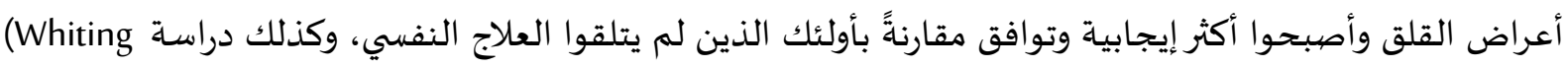
et al., 2020)

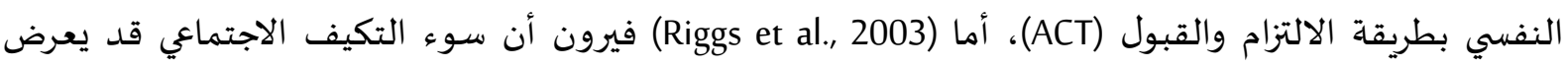

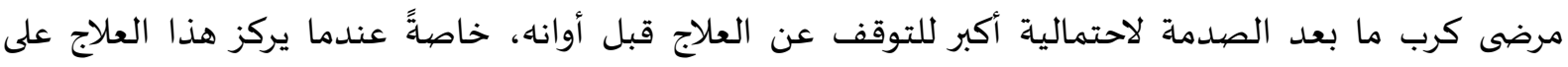
تخفيف أعراض اضطراب ما بعد الصدمة. ويرى معدوا هذه الدراسة أن التعرض للتحرش الجنسي وخصوصاً في مراحل الطفولة الأولى يعتبر نوعاً من

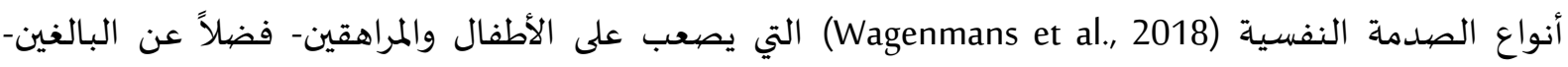
تجاوزها أو التعافي من آثارها بشكل فردي، وذلك لجهلهم بطرق التعامل والعلاج المناسبة والتي تتوفر في العيادات

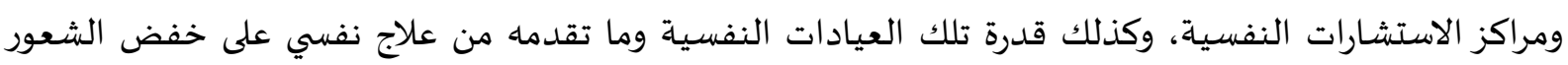

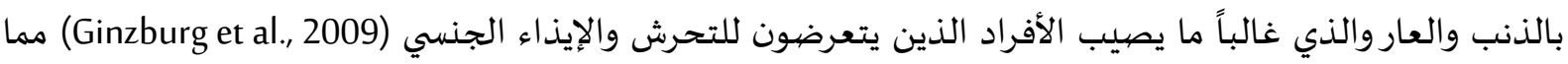
ينعكس على تقديرهم لذواتهم وتوافقهم مع مجتمعههم، وغالباً ما يكون سبب تلك المشاعر السلبية هو تبني الأفراد 
لبعض التشوهات المعرفية وأخطاء التفكير الناتجة عن الصدمة النفسية والتي تقودهم لاستنتاجات خاطئة حول دورهم في الأحداث الصادمة (Kubany \& Manke, 1995).

مناقشـة الفرض الثاني: توجد فروق بين المراجعين وغير المراجعين للعيادة النفسية بعد التعرض للتحرش الجنسي في تقدير الذات.

وفي ذات السياق، أسفرت نتائج التحليل الاحصائي عن حصول المراجعين للعيادة النفسية بعد التعرض الته لخبرة للتحرش الجنسي على متوسط درجات أعلى من غير المراجعين وذلك على مقياس تقدير الذات، وتتفق نتيجة

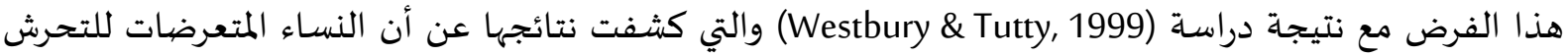

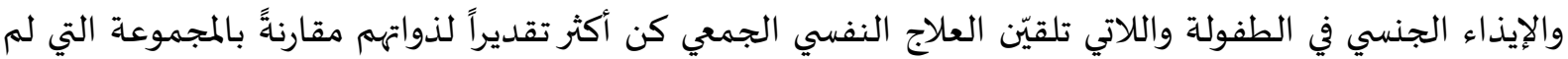

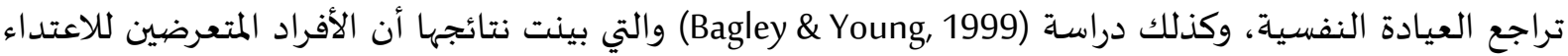

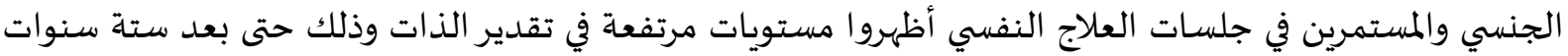

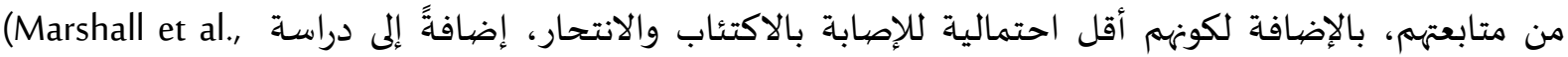
(1997 والتي خلصت نتائجها إلى ارتباط برامج العلاج النفسي القائم على تعزيز تقدير الذات لدى الأطفال المعنفون جنسياً بانخفاض الشعور بالوحدة وتحسن مشاعر الألفة والمودة، في حين أوضحت دراسة (Murphy et al., 2005) أن متابعة الرجال العنيفين لبرنامج علاجي مصهمم لتعزيز التعاطف مع الذات والآخرين وكذلك جلسات التهات العلاج النفسي السلوكي أدت لتحسن جوهري وملحوظ في تقديرهم لذواتهم مقارنةً بما كانوا عليه قبل تلقي العلاج. في الوقت الذي بحثت فيه دراسة (Sánchez- Meca et al., 2011) والقائمة على مراجعة 33 دراسة Meta- analysis عن لنوفي مدى فعالية العلاج النفسي للأطفال والمراهقين المتعرضين للاعتداء الجنسي في رفع تقدير الذات وخفض القلق والاكتئاب، والتي كشفت نتائجها عن أن الأفراد الذين تلقوا العلاج المعرفي السلوكي إلى جانب العلاج بالمساندة

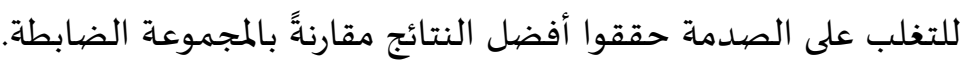
ويرجع كاتبو هذه السطور السبب في ذلك إلى أن أثر الصدمة النفسية والإيذاء والتحرش الجنسي لا يقتصر على التوافق الاجتماعي للفرد فحسب، بل إن أثره يمتد إلى الإضرار بتقدير الأفراد لذواتههم ومدى تأثر نظرتههم

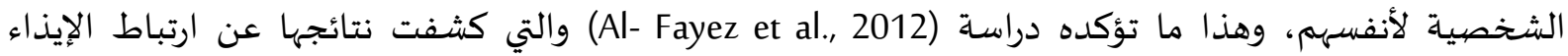

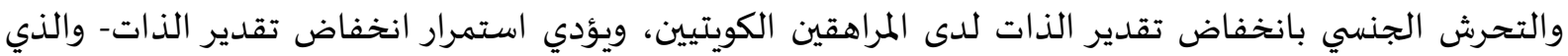

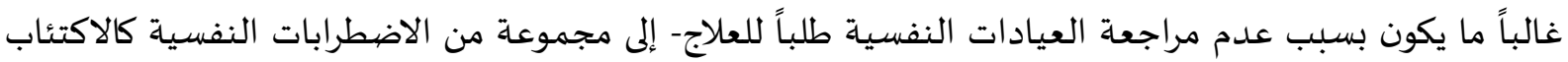

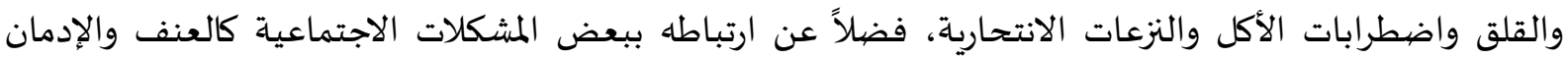

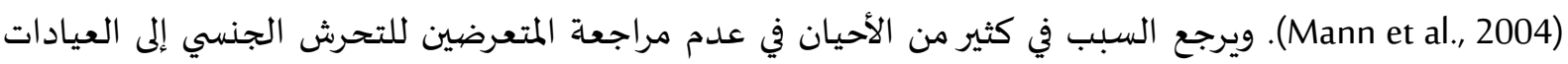

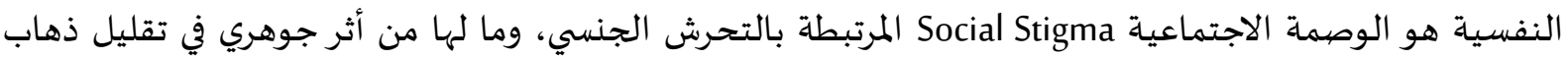

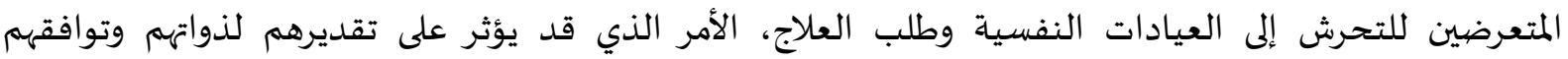
اجتماعياً أثناء رحلة تعافيهم من أثر الصيدمة النفسية (Kennedy \& Prock, 2018).

مناقشَة الفرض الثالث: توجد علاقة ارتباطية بين التوافق الاجتماعي وتقدير الذات لدى العينة الكلية

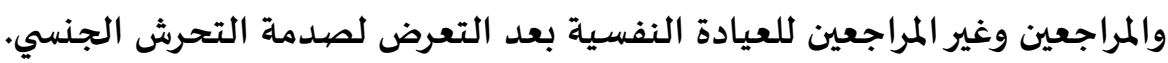

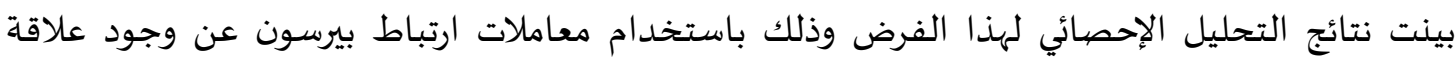
ارتباطية دالة وجوهرية بين التوافق الاجتماعي وتقدير الذات لدى المراجعين وغير المراجعين للعيادة النفسية والعينة العينة

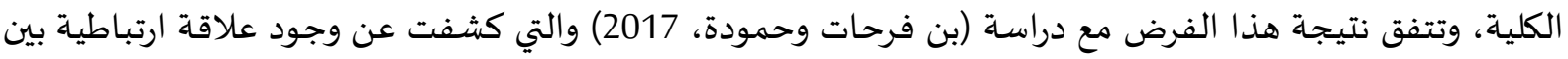


التوافق النفسي وتقدير الذات لدى عينة من طلبة الجامعة، وكذلك دراسة (Friedlander et al., 2007) والتي دلت

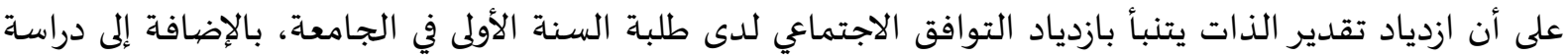
(Afolabi, 2014) الاجتماعي. في الوقت الذي أشارت فيه دراسة (Pasha \& Munaf, 2013) لوجود علاقة إيجابية بين تقدير الذات والتوافق الكلي وكذلك بعض أنواع التوافق الفرعية. ويرى الباحثون أن السبب قد يرجع في ذلك إلى أنه قد يتولد لدى الأفراد ذوو تقدير الذات المات المرتفع قدرات توافقية في نواحي الحياة الاجتماعية، وفي نفس الوقت فإن ازدياد قدرات الفرد على التوافق في الحياة بشكل عام قد يدل على نظرة واقعية وجيدة للفرد عن نفسه وهي أحد علامات تقدير الذات المرتفع (U. D.o \& Njoku, 2014). قائمة المراجع

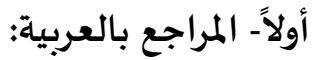
- باكيني، حكيمة؛ رمضاني، سارة (2017). تقدير الذات وعلاقته بالتوافق النفسي لدى المراهق الموهوب. رسالة

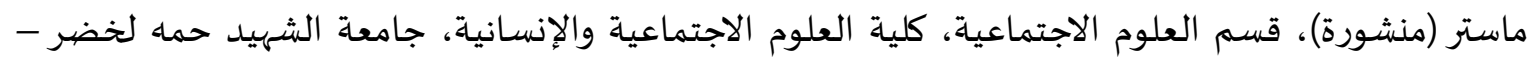
الوادي.

بن فرحات، آمنة؛ حمودة، وفاء (2017). علاقة تقدير الذات بالتوافق النفسي لدى الطالب الجامعي. رسالة

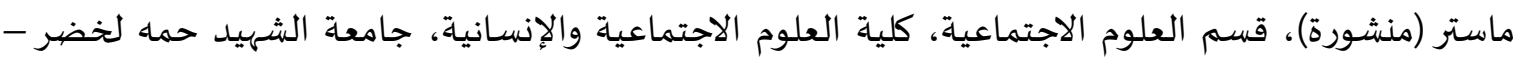
الوادي. - خفاجي، فاطمة (2008). ملاحظات أولية حول جرائم التحرش الجنسي في مصر. متوفر عبر هذا الرابط http://www.cihrs.org/Arabic/NewsSystem/Printable/Articles/1360 زهران، حامد عبد السلام (1997). الصحة النفسية والعلاج النفسي. عالم الكتب، القاهرة. سرى، إجلال محمد (1986). التوافق مع الاسم وعلاقته بالتوافق النفسي لدى الجنسين. دراسات تربوية، مج

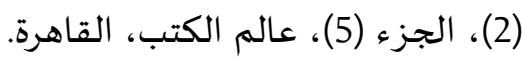

- - شاذلي، عبد الحميد محمد (2001). التوافق النفسي للمسنين. المكتبة الجامعية، الإسكندرية.

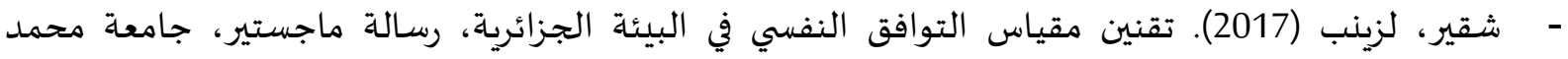
بوضياف، الجزائر.

- ـ صبرة، محمد علي؛ شريت، أشرف محمد (2004). الصحة النفسية والتوافق النفسي. دار المعرفة الجامعية، | الإسكندرية.

- عبيد، احمد، (2008). أفكار حول التحرش الجنسي في مصر. الحوار المتمدن، متوفر عبر هذا الرابط 2494 http://www.ahewar.org/debat/show.art.asp?aid=156254 - لمين، كورروغلي (2010). مساهمة في دراسة محاولة الانتحار عند المراهق بعد تعرضها لصدمة فشل. رسالة ماجستير، جامعة منتوري- قسطنطينة، الجزائر. 
- Abu- Hassan, W., \& Hamed, G. G. (2016). Post- Traumatic Stress Disorder and its Psychosocial Impacts on the Palestinian Child. The Canadian Journal for Middle East Studies, 1(2), 1142.

- Afolabi, O. A. (2014). Do Self Esteem and Family Relations Predict Prosocial Behaviour and Social Adjustment of Fresh Students? Higher Education of Social Science, 7(1), 26-34. https://doi.org/10.3968/5127

- Al- Fayez, G. A., Ohaeri, J. U., \& Gado, O. M. (2012). Prevalence of physical, psychological, and sexual abuse among a nationwide sample of Arab high school students: Association with family characteristics, anxiety, depression, self- esteem, and quality of life. Social Psychiatry and Psychiatric Epidemiology, 47(1), 53-66. https://doi.org/10.1007/s00127-010-0311- 2

- Bagley, C., \& Young, L. (1999). Long- term evaluation of group counselling for women with a history of child sexual abuse: Focus on depression, self- esteem, suicidal behaviours and social support. Social Work With Groups, 21(3), 63-73. https://doi.org/10.1300/J009v21n03_06

- Barnum, E. L., \& Perrone- McGovern, K. M. (2017). Attachment, self- esteem and subjective wellbeing among survivors of childhood sexual trauma. Journal of Mental Health Counseling, 39(1), 3955.

- British Crime Survey(2002). Rape and Sexual Assault of Women: Findings from the British Crime Survey. Home Office Research Study 159:1-6

- Chivers- Wilson K. A. (2006). Sexual assault and posttraumatic stress disorder: a review of the biological, psychological and sociological factors and treatments. McGill journal of medicine: MJM: an international forum for the advancement of medical sciences by students, 9(2), 111-118.

- Çelikel A, Demirkiran DS, Özsoy S, Zeren C, Arslan MM (2015) Factors Associated with PTSD in Cases of Sexual Assault. J Psychiatry 18: 181. doi:10.4172/2378- 5756.1000181

- Davidson JRT(2002). Pharmacotherapy of Posttraumatic Stress Disorder: Treatment Options, LongTerm follow- Up, and Predictors of Outcome. J Clin Psychiatry. 2002;61(5):52-56.

- De La Rosa, S., \& Riva, M. T. (2021). Relationship Variables in Group Psychotherapy for Women Sexual Trauma Survivors. International Journal of Group Psychotherapy, 71(1), 144- 179.

- Diagnostic and Statistical Manual of Mental Disorders (DSM- 5). (2013) American Psychiatric Association.

- Dunmore E, Clark DM, Ehlers A. Cognitive Factors Involved in the Onset and Maintenance of Post Traumatic Stress Disorder (PTSD) after Physical or Sexual Assault. Behaviour Research and Therapy. 1999; 37:809-829. [PubMed] [Google Scholar]

- Finkelhor, D., \& Browne, A. (1985). The traumatic impact of CSA: A conceptualization. American Journal of Orthopsychiatry, 55, 530- 541. 
- Friedlander, L. J., Reid, G. J., Shupak, N., \& Cribbie, R. (2007). Social Support, Self- Esteem, and Stress as Predictors of Adjustment to University Among First- Year Undergraduates. Journal of College Student Development, 48(3), 259-274. https://doi.org/10.1353/csd.2007.0024

- Ginzburg, K., Butler, L., Giese- Davis, J., Cavanaugh, C., Neri, E., Koopman, C., Classen, C., \& Spiegel, D. (2009). Shame, Guilt, and Posttraumatic Stress Disorder in Adult Survivors of Childhood Sexual Abuse at Risk for Human Immunodeficiency Virus. The Journal of Nervous and Mental Disease, 197, 536542. https://doi.org/10.1097/NMD.0b013e3181ab2ebd

- Haviland MG, Banta JE, Sonne JL, Przekop P. Posttraumatic Stress Disorder- Related Hospitalizations in the United States (2002-2011): Rates, Co- Occurring Illnesses, Suicidal Ideation/Self- Harm, and Hospital Charges. The Journal of nervous and mental disease. 2016; 204(2):78-86.

- Jaberghaderi, N., Greenwald, R., Rubin, A., Zand, S. O., \& Dolatabadi, S. (2004). A Comparison of CBT and EMDR for Sexually- abused Iranian Girls. Clinical Psychology \& Psychotherapy, 11(5), 358-368. https://doi.org/10.1002/cpp.395

- Johnson- Kwochka, A., Minor, K. S., Ashburn- Nardo, L., Wu, W., Stull, L. G., \& Salyers, M. P. (2021). A new look at the attribution model: Considerations for the measurement of public mental illness stigma.

- Kachadourian L, Harpaz- Rotem I, Tsai J, Southwick S, Pietrzak RH.(2021).Mindfulness as a mediator between trauma exposure and mental health outcomes: Results from the National Health and Resilience in Veterans Study. Psychological Trauma: Theory, Research, Practice and Policy. Jan.

- Kennedy, A., \& Prock, K. (2018). "I Still Feel Like I Am Not Normal'”: A Review of the Role of Stigma and Stigmatization Among Female Survivors of Child Sexual Abuse, Sexual Assault, and Intimate Partner Violence. Trauma Violence \& Abuse, 19. https://doi.org/10.1177/1524838016673601

- Koss MP, Figuerdo AJ. Change in Cognitive Mediators of Rape's Impact on Psychosocial Health Across 2 Years of Recovery. Journal of Consulting and Clinical Psychology. 2004;72(6):10631072. [PubMed] [Google Scholar]

- Kubany, E. S., \& Manke, F. P. (1995). Cognitive therapy for trauma- related guilt: Conceptual bases and treatment outlines. Cognitive and Behavioral Practice, 2(1), 27-61. https://doi.org/10.1016/S10777229(05)80004- 5 .

- Kucharska, J. (2017). Sexual and non-sexual trauma, depression and self-esteem in a sample of polish women. A cross-sectional study. Clinical Psychology \& Psychotherapy, 24(1), 186- 194.

- Mann, M. (Michelle), Hosman, C. M. H., Schaalma, H. P., \& de Vries, N. K. (2004). Self- esteem in a broad- spectrum approach for mental health promotion. Health Education Research, 19(4), 357-372. https://doi.org/10.1093/her/cyg041 
- Marshall, W. L., Champagne, F., Sturgeon, C., \& Bryce, P. (1997). Increasing the self- esteem of child molesters. Sexual Abuse: A Journal of Research and Treatment, 9(4), 321-333. https://doi.org/10.1007/BF02674856

- Martin, J. D. (2000): Social psychology, North western university.

- Marylene Cloitre, Regina Miranda, K. Chase Stovall- McClough, Hyemee Han. (2005). Beyond PTSD: Emotion regulation and interpersonal problems as predictors of functional impairment in survivors of childhood abuse,Behavior Therapy,Volume 36, Issue 2,2005,Pages 119-124,ISSN 0005- 7894,

- Monson, C.M., Macdonald, A., Vorstenbosch, V., Shnaider, P., Goldstein, E.S.R., Ferrier-Auerbach, A.G. and Mocciola, K.E. (2012), Changes in Social Adjustment with Cognitive Processing Therapy: Effects of Treatment and Association with PTSD Symptom Change. JOURNAL OF TRAUMATIC STRESS, 25: 519526. https://doi.org/10.1002/jts.21735

- Murphy, C. M., Stosny, S., \& Morrel, T. M. (2005). Change in Self- Esteem and Physical Aggression During Treatment for Partner Violent Men. Journal of Family Violence, 20(4), 201. https://doi.org/10.1007/s10896-005-5983-0

- Pasha, H. S., \& Munaf, S. (2013). Relationship of Self- esteem and Adjustment in Traditional University Students. Procedia- Social and Behavioral Sciences, 84, 999-1004. https://doi.org/10.1016/j.sbspro.2013.06.688

- Pizarro Obaid F. (2012). Sigmund Freud and Otto Rank: debates and confrontations about anxiety and birth. Int J Psychoanal. 2012 Jun;93(3):693- 715. doi: 10.1111/j.1745- 8315.2012.00594.x. Epub 2012 Apr 16. Erratum in: Int J Psychoanal. 2012 Jun;93(3):i- ii. PMID: 22671256.

- Pretorius, G., \& Pfeifer, N. (2010). Group art therapy with sexually abused girls. South African Journal of Psychology, 4O(1), 63- 73.

- Riggs, D. S., Rukstalis, M., Volpicelli, J. R., Kalmanson, D., \& Foa, E. B. (2003). Demographic and social adjustment characteristics of patients with comorbid posttraumatic stress disorder and alcohol dependence: Potential pitfalls to PTSD treatment. Addictive Behaviors, 28(9), 1717-1730. https://doi.org/10.1016/j.addbeh.2003.08.044

- Rosenberg, M. (1965). Rosenberg self- esteem scale (RSE). Acceptance and commitment therapy. Measures package, 61(52), 18.

- Robinaugh, D.J. and McNally, R.J. (2011), Trauma centrality and PTSD symptom severity in adult survivors of childhood sexual abuse. J. Traum. Stress, 24: 483- 486. https://doi.org/10.1002/jts.20656

- Sánchez- Meca, J., Rosa- Alcázar, A. I., \& López- Soler, C. (2011). The psychological treatment of sexual abuse in children and adolescents: A meta- analysis. International Journal of Clinical and Health Psychology, 11(1), 67-93.

- Santarnecchi, E., Bossini, L., Vatti, G., Fagiolini, A., La Porta, P., Di Lorenzo, G., Siracusano, A., Rossi, S., \& Rossi, A. (2019). Psychological and Brain Connectivity Changes Following Trauma- Focused CBT 
and EMDR Treatment in Single- Episode PTSD Patients. Frontiers in Psychology, 10. https://doi.org/10.3389/fpsyg.2019.00129.

- Sharma, Mohit. (2019). Self- esteem of incoming and outgoing students of the college. International Journal for Research in Applied Science \& Engineering Technology (IJRASET) ISSN: 2321- 9653; IC Value: 45.98; SJ Impact Factor: 6.887 Volume 7 Issue IV, Apr 2019- Available at www.ijraset.com

- Smith, E. R.; Mackie, D. M. (2007). Social Psychology (Third ed.). Hove: Psychology Press. ISBN 978- 184169- 408- 5.

- Shapiro F. (2002). EMDR as an Integrative Psychotherapy Approach: Experts of Diverse Orientations Explore the Paradigm Prism. Washington, DC: American Psychological Association Books;.

- U. D.o, B., \& Njoku, J. (2014). The Role of the Teacher in Improving Students Self Esteem. International Journal of Academic Research in Progressive Education and Development, 3(1), 47-53.

- Van Bruggen LK, Runtz MG, Kadlec H. (2006). Sexual revictimization: the role of sexual self- esteem and dysfunctional sexual behaviors. Child Maltreat. 2006 May;11(2):131- 45. doi: 10.1177/1077559505285780. PMID: 16595847.

- Wagenmans, A., Minnen, A. V., Sleijpen, M., \& Jongh, A. D. (2018). The impact of childhood sexual abuse on the outcome of intensive trauma- focused treatment for PTSD. European Journal of Psychotraumatology, 9(1), 1430962. https://doi.org/10.1080/20008198.2018.1430962

- Walsh, K., Danielson, C. K., McCauley, J. L., Saunders, B. E., Kilpatrick, D. G., \& Resnick, H. S. (2012). National prevalence of posttraumatic stress disorder among sexually revictimized adolescent, college, and adult household- residing women. Archives of general psychiatry,69(9), 935-942. https://doi.org/10.1001/archgenpsychiatry.2012.132

- Walter, O. (2020). Can participation in belly dancing improve body image and self- esteem in women who have experienced sexual harassment?. Journal of Aggression, Maltreatment \& Trauma, 29(6), 748- 765.

- Weiten, W. (1998). Psychology: Themes and variations. Pacific Grove, CA: Brooks/ Cole Publishing Co.

- Westbury, E., \& Tutty, L. M. (1999). The efficacy of group treatment for survivors of childhood abuse. Child Abuse \& Neglect, 23(1), 31- 44.

- Whiting, D., Deane, F., McLeod, H., Ciarrochi, J., \& Simpson, G. (2020). Can acceptance and commitment therapy facilitate psychological adjustment after a severe traumatic brain injury? A pilot randomized controlled trial. Neuropsychological Rehabilitation, 30(7), 1348-1371. https://doi.org/10.1080/09602011.2019.1583582

- WILSON, S. A., BECKER, L. A., \& TINKER, R. H. (1995). Eye movement desensitization and reprocessing (EMDR) treatment for psychologically traumatized individuals. Eye Movement Desensitization and Reprocessing (EMDR) Treatment for Psychologically Traumatized Individuals, 63(6), 928-937. 
- Wright, L., \& Warner, A. (2020). EMDR treatment of childhood sexual abuse for a child molester: selfreported changes in sexual arousal. Journal of EMDR practice and research.

- Zeanah, P. D., \& Schwarz, J. C. (1996). Reliability and validity of the Sexual Self- Esteem InventoryWomen. Assessment, 3, 1- 15. 